\title{
可见光促进烷基硼化合物转化研究进展
}

\author{
史敦发 ${ }^{a, b}$ 王 露 ${ }^{a}$ 夏春谷 ${ }^{a}$ 刘 超*,a \\ ( ${ }^{a}$ 中国科学院兰州化学物理研究所 羰基合成与选择氧化国家重点实验室 兰州 730000) \\ $\left({ }^{b}\right.$ 中国科学院大学 北京 100049)
}

\begin{abstract}
摘要 有机硼化合物来源广泛, 转化丰富多样, 已经成为一种重要的有机合成中间体, 被广泛地应用于医药、农药以及 有机光电材料的合成中. 烷基嗍化合物作为一类重要的有机硼化合物, 其合成以及转化研究具有重要意义. 可见光近 来在有机反应领域受到广泛关注. 在可见光诱导下, 四配位烷基硼化合物能发生单电子转移产生烷基自由基，从而进 一步构建不同的化学键. 综述了该研究领域的相关进展.
\end{abstract}

关键词 硼; 光催化; 自由基; 单电子转移

\section{Recent Advances in Visible-Light-Promoted Transformation of Alkyl Boron Compounds}

\author{
Shi, Dunfa ${ }^{a, b}$ Wang, $\mathrm{Lu}^{a} \quad \mathrm{Xia}$, Chungu ${ }^{a} \quad$ Liu, Chao ${ }^{*, a}$ \\ ( ${ }^{a}$ State Key Laboratory for Oxo Synthesis and Selective Oxidation, Lanzhou Institute of Chemical Physics, \\ Chinese Academy of Sciences, Lanzhou 730000) \\ ( ${ }^{b}$ University of Chinese Academy of Sciences, Beijing 100049)
}

\begin{abstract}
Organoboron compounds are valuable synthetic intermediates and widely used in the synthesis of medicine, pesticide and organic optoelectronic materials due to their extensive resouce and highly transformable ability. Among various organoboron compounds, the synthesis and transformation of alkylboron compounds have attracted much attention. As a sustainable and green energy, visible light shows an important effect in organic systhesis. Tetracoordinated alkylboron compounds could occur single electron transfer (SET) process to generate alkyl radical for further transformations. Herein, the recent advances in the photoinduced transformation of alkyl boron compounds are summarized.
\end{abstract}

Keywords boron; photocatalysis; radical; single electron transfer

有机嗍化合物作为重要的有机合成中间体，被广泛 地应用于医药、农药、配体以及有机光电材料的合成等 领域中. 一直以来, 其合成与转化受到广泛的关注，其 中部分反应因为巨大的应用价值已经成为人名反应，如 Suzuki-Miyaura 交叉偶联反应 ${ }^{[1]}$ 、Brown 嗍氢化反应 ${ }^{[2]}$ 、 Chan-Lam 反应 ${ }^{[3]}$ 以及 Zweifel 烯基化反应 ${ }^{[4]}$ 等.

在各类有机硼化合物中，烷基硼化合物因其合成方 法丰富及结构多样而一直受到化学家们的高度关注与 重视. 过渡金属催化的 Suzuki-Miyaura 偶联反应主要实 现了芳基硼类化合物的转化 ${ }^{[5]}$. 对于烷基硼而言, 因碳
硼键极性较小, 在金属催化的偶联反应中难以进行转金 属化过程并且存在潜在的 $\beta$-氢消除副反应. 因此，尽管 目前有少量的报道，金属催化烷基硼的偶联反应仍具有 很大的挑战, 基于四配位嗍 1,2-迁移反应已经成为烷基 硼转化的重要方法 ${ }^{[6]}$. 近些年来, 自由基化学得到迅速 的发展，并成为了一类前景良好并且具有潜在普适性的 有机合成方法. 可见光作为一种清洁可持续能源, 其参 与高效的自由基引发体系不仅具有优异的经济性，也符 合绿色化学的发展潮流 ${ }^{[7]}$. 目前从反应机理上看, 光催 化烷基硼化合物脱硼生成烷基自由基模式主要为两类:

\footnotetext{
* Corresponding author. E-mail: chaoliu@licp.cas.cn

Received June 18, 2020; revised August 29, 2020; published online September 8, 2020.

Dedicated to the 40th anniversary of Chinese Journal of Organic Chemistry.

Project supported by the National Natural Science Foundation of China (Nos. 21673261, 91745110, 21872156), the Natural Science Foundation of Jiangsu Province (Nos. BK20190002, BK20181194) and the Youth Innovation Promotion Association of the Chinese Academy of Sciences (No. 2018458).

国家自然科学基金(Nos. 21673261，91745110，21872156)、江苏省自然科学基金(Nos. BK20190002，BK20181194)和中国科学院青年创新促进会(No. 2018458)资助项目.
} 
(1)烷基四配位硼化合物被激发态的光催化剂单电子氧 化释放出烷基自由基以及三配位硣(Scheme 1, a); (2)三 配位硼化合物在外加自由基作用下，发生自由基交换， 释放出烷基自由基(Scheme 1, b). 生成的烷基自由基随 后被不同的自由基受体捕获，进一步转化形成不同的化 学键. 2015 年, Blanchard 和 Bisseret 等 ${ }^{[8]}$ 综述了光催化下 有机硼化合物的合成以及转化的相关研究. 2018 年, Li, Liu 和 Yan 等 ${ }^{[9]}$ 共同综述了烷基硼化合物的自由基转化, 介绍了氧化剂介导下烷基嗍化合物的转化以及可见光 催化下烷基三氟嗍酸钾在 $\mathrm{C}-\mathrm{C}$ 成键上的应用. 尽管上 述文献中从不同角度包含了可见光催化烷基硼化合物 的部分转化反应, 但是我们注意到目前光催化烷基嗍化 合物的转化尚无系统的总结. 基于此, 本文从成键类型 包括碳碳成键、碳氧成键、碳硫成键以及碳氢成键出发, 综述烷基硼化合物在可见光促进下脱硼衍生化的研究 进展.

(a)

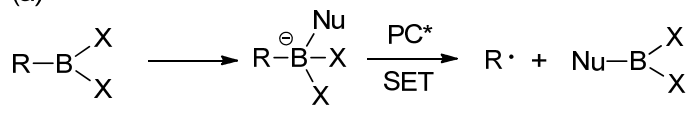

(b)

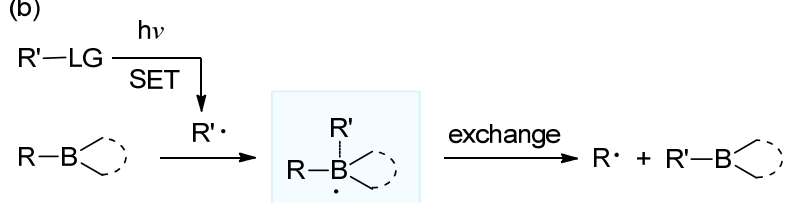

图式 1 光催化烷基嗍化合物的活化模式

Scheme 1 Activated models of alkyl boron compounds under photoredox condition

\section{$1 \mathrm{C}$ - C 成键反应}

$\mathrm{C}-\mathrm{C}$ 成键反应是目前有机合成研究最为广泛的反 应. 通过可见光催化介导烷基硼化合物脱硼来实现碳碳 键偶联, 往往具有条件温和及官能团兼容性广的优点.

\section{$1.1 \mathrm{C}\left(\mathrm{sp}^{3}\right)-\mathrm{C}(\mathrm{sp})$ 成键反应}

2014 年, 陈以昀课题组 ${ }^{[10]}$ 报道了首例光催化烷基 三氟硼酸钾的脱嗍炔基化反应，实现了烷基硼化合物脱 碍构建 $\mathrm{C}\left(\mathrm{sp}^{3}\right)-\mathrm{C}(\mathrm{sp})$ 键(Scheme 2). 该反应具有优异的 普适性, 不仅可以兼容一级、二级以及三级砋化合物, 同时烷基硼酸也反应良好. 此外, 该反应还可以兼容敏 感的官能团如卤素( $\mathrm{Br}, \mathrm{I})$ 、炔基、醛酮羰基以及羟基等. 值得一提的是, 该反应在中性水缓冲液中也可以进行, 并且向体系中添加氨基酸、核苷、低聚糖、核酸、蛋白 质和细胞裂解物均不会影响反应. 通过一系列控制实验 证明，三价的有机碘试剂 $\mathrm{BI}-\mathrm{OH}$ 释放出 $\mathrm{BI}$ 自由基，该自 由基氧化激发态 $\mathrm{Ru}(\mathrm{II})$ 生成 $\mathrm{Ru}(\mathrm{III})$ 引发了整个反应. 生 成的 $\mathrm{Ru}(\mathrm{III})$ 与烷基喼化合物(烷基三氟硼酸钾或者烷基
硼酸)发生单电子氧化生成烷基自由基以及初始 $\mathrm{Ru}(\mathrm{II})$ 光催剂. 随后烷基自由基与炔 $\mathrm{BI}$ 发生自由基加成并随 后消除得到目标产物，同时释放出氧化激发态 $\mathrm{Ru}(\mathrm{II})$ 的 BI 自由基实现了反应的循环.

$$
\begin{aligned}
& {\left[\mathrm{Ru}(\mathrm{bpy})_{3}\right]\left(\mathrm{PF}_{6}\right)_{2}(2 \mathrm{~mol} \%)} \\
& \mathrm{BI}-\mathrm{OH} \text { ( } 0.5 \text { equiv.) }
\end{aligned}
$$

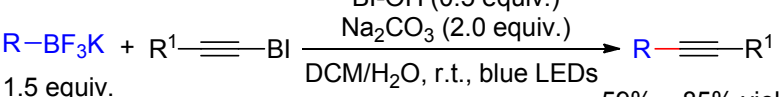

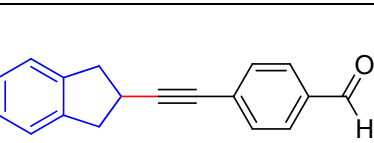

$81 \%$

$75 \%$

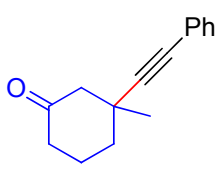

$60 \%$<smiles>CC(C)(C#CC#Cc1ccccc1)CBr</smiles>

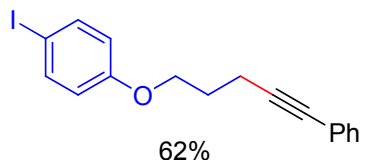

$62 \%$

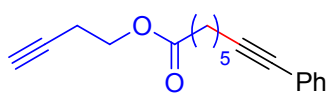

$66 \%$

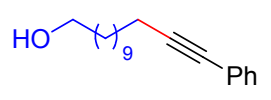

$65 \%$

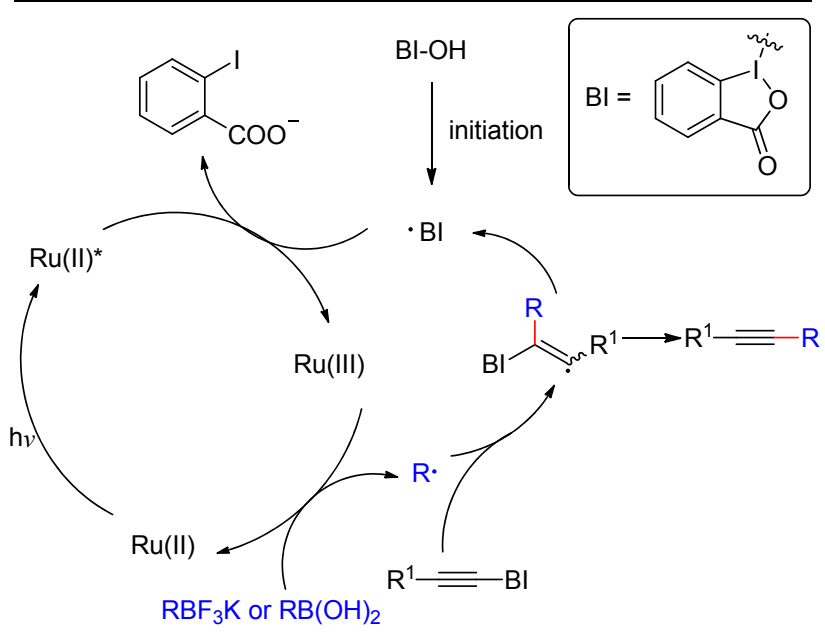

图式 2 光催化烷基三氟嗍酸钾的炔基化反应

Scheme 2 Photoredox catalyzed alkynylation of alkyltrifluoroborates

2016 年, 许华建课题组 ${ }^{[11]}$ 以 $\mathrm{Ru}(\mathrm{bpy})_{3}\left(\mathrm{PF}_{6}\right)_{2}$ 作为催 化剂, 对甲基苯磺酰腈( $\mathrm{TsCN}$ )作为㲵基来源, BI-OAc 和 三氟醋酸(TFA)作为添加剂, 实现了烷基三氟硼酸钾的 脱硼氰基化反应(Scheme 3, a). 该反应在空气中即可进 行, 条件温和. 结合烯烃嗍氢化反应, 作者通过三步以 $61 \%$ 的收率得到烯烃反马氏氰基化产物. 值得一提的 是，烷基频哪醇硼酸酯作为底物时，反应也能以 $30 \%$ 的 收率得到目标产物. 同年, Molander 课题组 ${ }^{[12]}$ 以 Mes$\mathrm{Acr}^{+}$作为光催化剂, $N, N$-二甲基甲酰胺(DMF)作为溶剂, 使用同样的氰源实现了一级以及二级烷基三氟硼酸钾 
的脱硼氰基化(Scheme 3, b). 相比较而言, 该反应更为 简单且不需要任何添加剂, 同时避免了过渡金属的使 用.

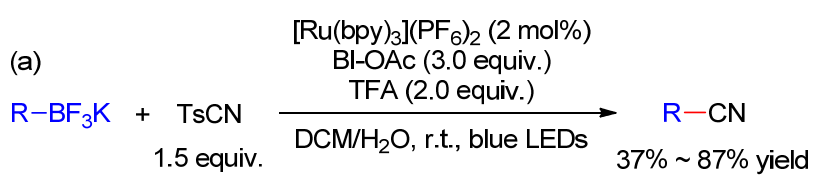

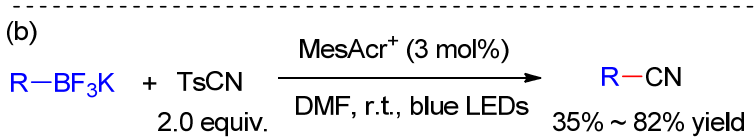

图式 3 光催化烷基三氟硼酸钾的氧基化反应

Scheme 3 Photoredox catalyzed cyanylation of alkyltrifluoroborates

近期, 刘超课题组 ${ }^{[13]}$ 报道了光催化的烷基频哪醇 嗍酸酯脱嗍炔基化反应. 他们以廉价易得的 $\mathrm{NaOMe}$ 或 者 $\mathrm{NaOH}$ 作为烷基硼酸酯的活化试剂, 使其成为可以被 激发态光催化剂单电子氧化的四配位嗍前体, 并通过核 磁 ${ }^{11} \mathrm{~B}$ 谱实验证明了四配位嗍中间体的形成. 进一步通 过自由基捕获实验、电子顺磁共振(EPR)实验以及苂光 淬灭实验证明了该四配位硼前体为自由基源. 该反应不 需要过渡金属，底物兼容性好. 此外，对于二硼化合物， 反应能选择性对位阻大的一侧碳嗍键进行炔基化 (Scheme 4).

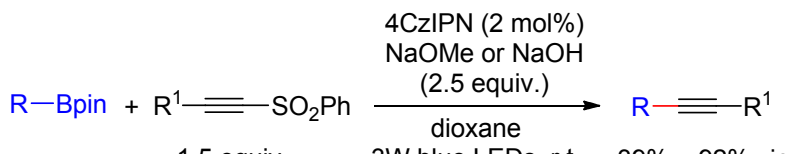

1.5 equiv. $\quad 3 \mathrm{~W}$ blue LEDs, r.t. $\quad 39 \% \sim 92 \%$ yield

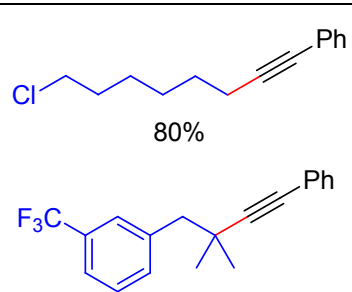

$54 \%$
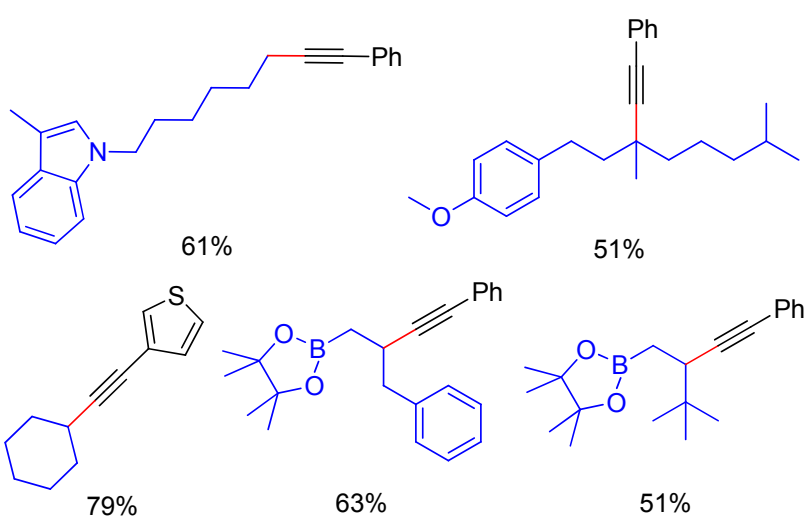

图式 4 光催化烷基硼酸酯的炔基化反应

Scheme 4 Photoinduced alkynylation of alkyl pinacol boronates

\section{$1.2 \mathrm{C}\left(\mathrm{sp}^{3}\right)-\mathrm{C}\left(\mathrm{sp}^{2}\right)$ 成键反应}

2014 年, Molander 课题组 ${ }^{[14]}$ 报道了首例光/镍协同 催化苄基三氟硼酸钾与芳基溴化物的偶联反应(Scheme 5，a). 相比于传统的过渡金属催化模式来说，该反应具 有反应条件温和及底物兼容性好的特点. 该反应可能的 机理为: 苄基三氟硼酸钾被激发态 Ir 光催化剂单电子氧 化为茮基自由基; 同时, Ir 光催化剂被还原生成还原态 Ir 催化剂, 还原态的 Ir 光催化剂将体系中 Ni(I)催化剂单 电子还原为 $\mathrm{Ni}(0)$ 物种; 进一步, 生成的 $\mathrm{Ni}(0)$ 物种与芳 基卤化物氧化加成得到芳基镍物种 $\mathbf{A}$ ，芳基镍物种 $\mathbf{A}$ 捕 捉体系中茮基自由基生成 $\mathrm{Ni}$ (III)中间体 $\mathbf{B}$ ，随后 $\mathrm{Ni}$ (III) 中间体 B 发生还原消除得到目标产物并生成初始 Ni(I) 物种. 此外, 当使用手性 Box 类配体时, 能以 $50 \%$ 的 ee 值得到目标产物，表明该转化可实现立体选择性控制
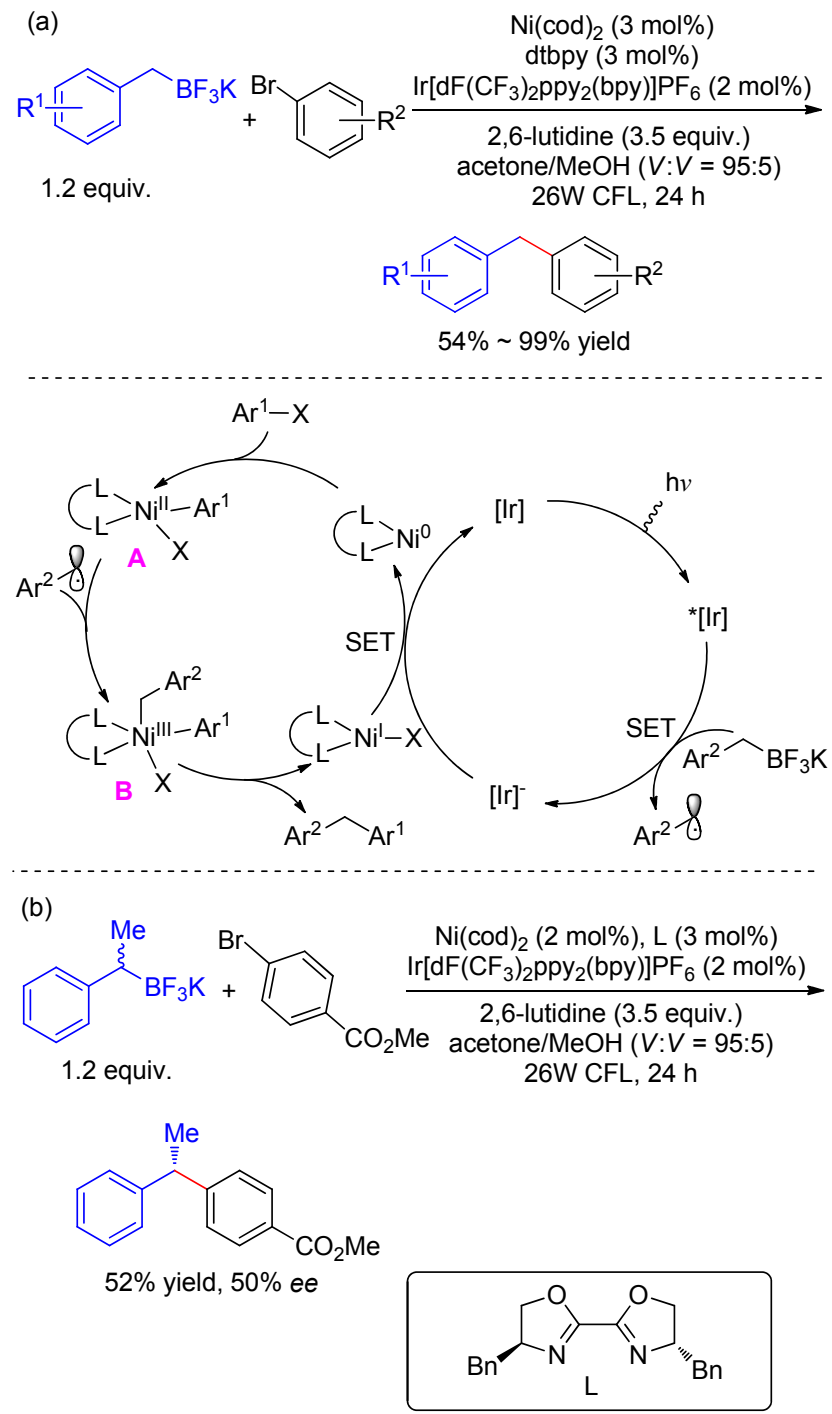

图式 5 光镍协同催化苄基三氟嗍酸钾的芳基化反应

Scheme 5 Photoredox/nickel dual catalyzed arylation of benzylic trifluoroborates 
(Scheme 5, b). 随后, Molander 小组与 Kozlowski 小组 ${ }^{[15]}$ 合作通过密度泛函理论(DFT)计算来进一步验证了该反 应的机理. 在此工作基础上, Molander 课题组进一步发 展了二级烷基三氟硼酸钾 ${ }^{[16]}$ 、三级烷基三氟硼酸钾 ${ }^{[17]}$ 的可见光镍协同催化的脱硼芳基化反应, 为烷基三氟硼 酸钾提供了一种条件温和且反应高效的芳基化策略. 该 策略还可被应用到 $\alpha$-羟基三氟硼酸钾 ${ }^{[18]} 、 \alpha$-三氟甲基三 氟硼酸钾 ${ }^{[19]} 、 \beta$-酰基三氟硼酸钾 ${ }^{[20]}$ 以及 $\alpha$-烷氧基三氟硼 酸钾 ${ }^{[21]}$ 的脱硼芳基化反应. 这些反应均表现出良好的 底物普适性(Scheme 6).

2016 年, Ley 课题组 ${ }^{[22]}$ 首次报道了流动光催化苄基
嗍酸酯脱硼芳基化的方法(Scheme 7). 反应机理研究表 明，吡啶类的 Lewis 碱如 4-二甲氨基吡啶(DMAP)等可 以与苄基硼酸酯形成四配位喼化合物，该四配位硣化合 物可被激发态的 Ir 催化剂单电子氧化生成茮基自由基. 进一步通过镍催化和芳基卤代烃偶联. 值得一提的是, 含氰基的氮杂芳烃既作为活化试剂又作为原料，同苄基 自由基发生脱氰基偶联反应并且不需要镍作为共催化 剂. 相较于的烷基三氟硼酸钾，烷基硼酸酯来源更加广 泛，更具有经济性.

Minisci 反应为碳自由基亲核加成到缺电子杂环上, 随后发生 $\mathrm{C}-\mathrm{H}$ 键取代反应 ${ }^{[23]}$. 光催化作为产生亲核自

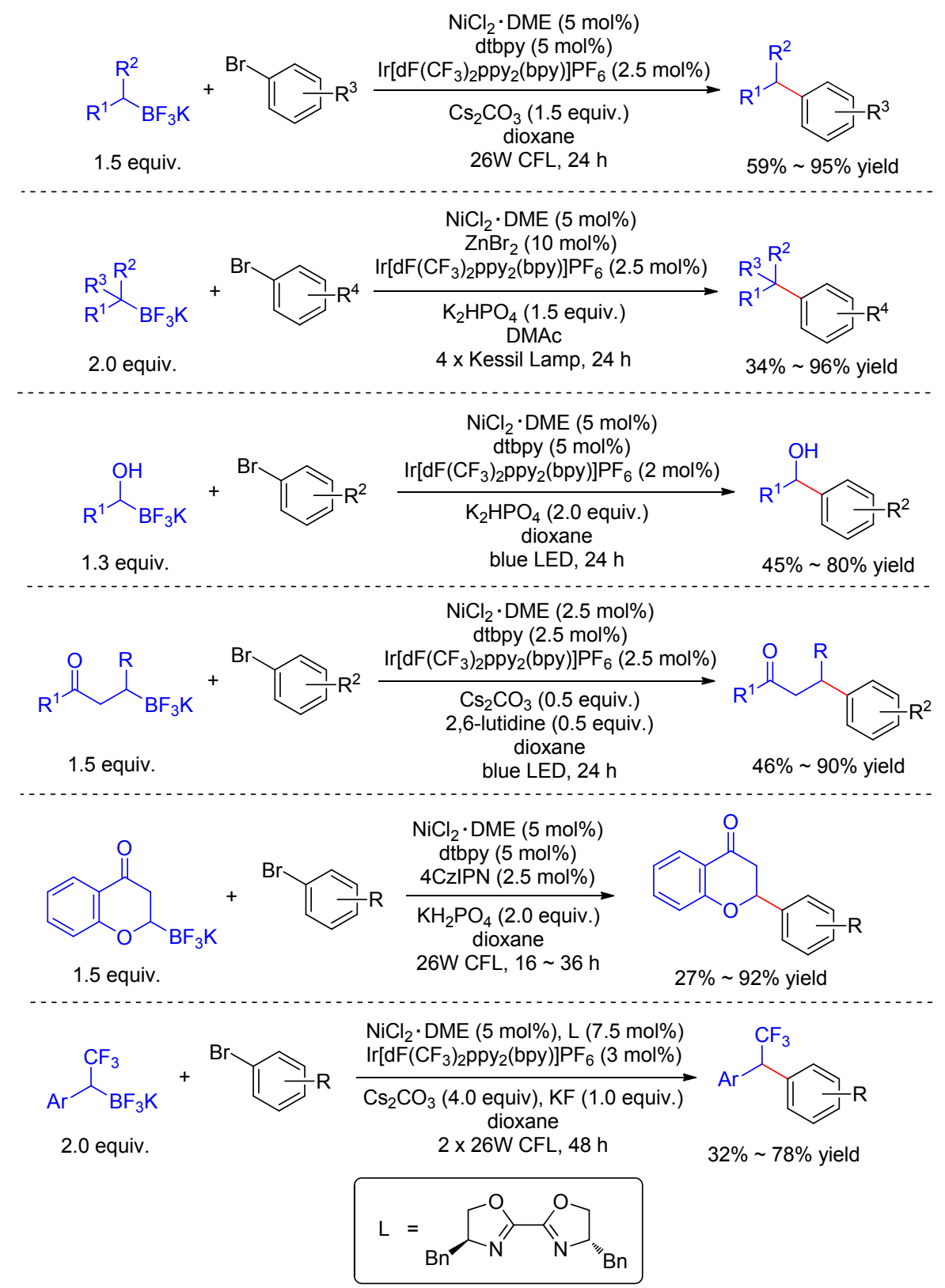

图式 6 光镍协同催化烷基三氟嗍酸钾的芳基化反应

Scheme 6 Dual photoredox/nickel catalyzed arylation of alkyltrifluoroborates 


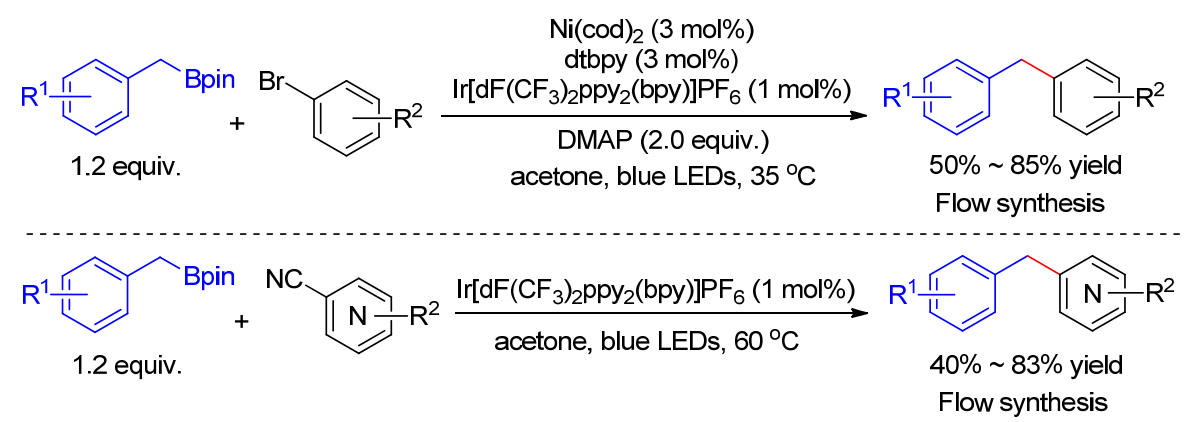

图式 7 光催化苄基频哪醇硼酸酯的芳基化反应

Scheme 7 Photoredox catalyzed arylation of benzylic pinacol boronates

由基的一种强有力的手段已经被应用于该反应 ${ }^{[24]}$. 作 为一种化学性质稳定并且来源广泛的自由基源, 烷基硼 化合物的 Minisci 反应用于含氮杂环化合物衍生化的反 应成为研究的重点. 2016 年, 陈弓课题组 ${ }^{[25]}$ 报道了以 $\mathrm{Ru}(\mathrm{bpy})_{3} \mathrm{Cl}_{2}$ 作为光催化剂, 一级以及二级烷基硼酸作为 烷基源的光催化含氮杂环芳烃碳氢键烷基化反应 (Scheme 8). 该反应具有优异的底物兼容性和选择性, 可以实现如奎宁、咖啡因、喜树碱、泛昔洛韦、法舒地 尔等药物分子或者天然产物定点烷基化, 为药物后续开 发提供了可能. 实验以及 DFT 计算表明该反应的关键 步骤为 BI-OAc 被激发态的 $\mathrm{Ru}(\mathrm{II})$ 还原成邻碘苯甲酰氧 自由基, 该自由基具有亲嗍的性质, 可以和烷基硼酸发 生反应生成烷基自由基. 随后烷基自由基对含氮杂环发 生亲核加成, 进一步芳构化得到目标产物。

2017 年, 许华建课题组 ${ }^{[26]}$ 以 $\mathrm{Ru}$ 配合物作为光催化 剂, 三氟乙酸作为添加剂, BI-OAc 作为氧化剂实现了光 催化吡啶氮氧化物 $\mathrm{C}(2)$ 位碳氢键的烷基化反应(Scheme 9 , a). 该反应底物普适性好，可兼容酯、酰胺、醚、氰基、 酮、炔烃和卤化物等. 通过该策略，他们以三步 $59 \%$ 的 收率合成药物分子环吡酮. 2017 年, Molander 课题组 ${ }^{[27]}$ 报道了无金属光催化的烷基三氟硼酸钾作为自由基源 的 Minisci 反应(Scheme 9, b). 他们用此方法实现了温和 条件下菲啰啉配体以及联吡啶配体高区域选择性高收 率烷基化反应. 2019 年, 徐海超课题组 ${ }^{[28]}$ 采用光电共催 化的策略也同样实现了烷基三氟硼酸钾的 Minisci 反应 (Scheme 9, c). 相比较而言, 该反应不需要外加氧化剂 并且可以兼容各类一级、二级以及三级烷基三氟硼酸钾. 此外, 他们通过该方法对伏立康唑、喜树碱、法舒地尔 和奎宁进行了选择性烷基化.

2016年, Molander 课题组 ${ }^{[29]}$ 报道了可见光镍协同催 化 $\alpha$ 烷氧基三氟硼酸钾的脱硼酰基化反应. 该反应机理 为: 酰氯和金属镍形成酰基镍物种, 随后与烷氧基三氟 硼酸钾脱硼生成的烷氧基自由基发生氧化加成，最后还 原消除生成目标产物。该策略发展了一种新的酰基成键 模式. 随后他们进一步拓展了该活化模式的普适性, 分
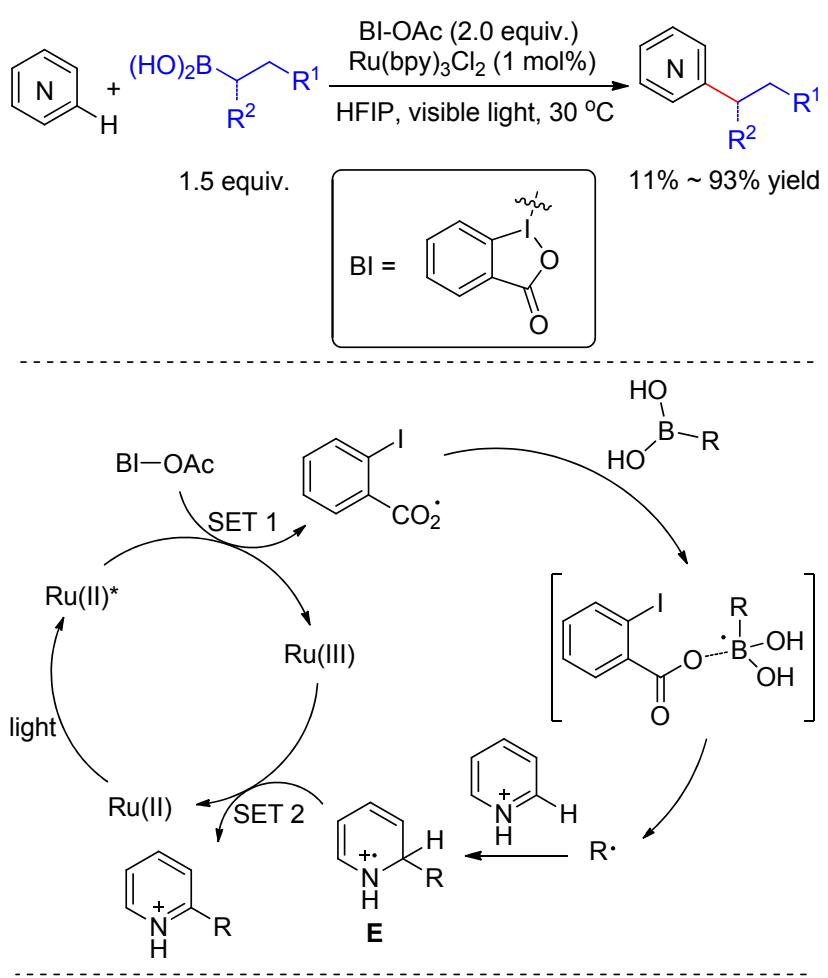<smiles>C=CC1CN2CCC1C2[C@H](O)c1cc(C2CCCCC2)nc2ccc(OC)cc12</smiles>

quinine, $90 \%$<smiles>CC(=O)OCC(CCn1cnc2c(C3CCCCC3)nc(N)nc21)COC(C)=O</smiles>

famciclovir, $50 \%$

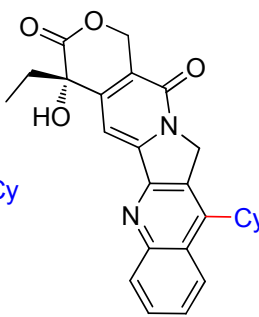

camptothecin, $91 \%$<smiles>CCc1nccc2c(S(=O)(=O)N3CCCNCC3)cccc12</smiles>

Fasudil, 61\%
图式 8 光催化烷基嗍酸的 Minisci 反应

Scheme 8 Photoredox catalyzed Minisci reaction of alkyl boric acid 


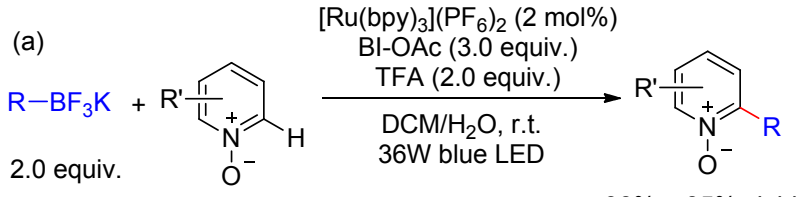

$38 \% \sim 85 \%$ yield

(b)

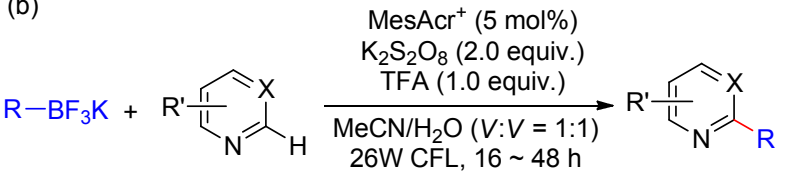

1.0 equiv.

$11 \% \sim 95 \%$ yield

(c)

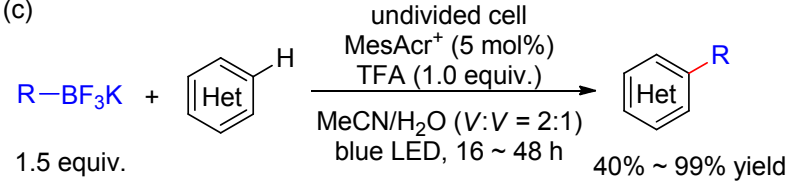

图式 9 光催化烷基三氟硼酸钾的 Minisci 反应

Scheme 9 Photoredox catalyzed Minisci reaction of alkyltrifluoroborates

别实现了在可见光金属镍共催化条件下，烷基三氟硼酸 钾与酰氯 ${ }^{[30]}$ 、酰胺 ${ }^{[31]}$ 及羧酸 ${ }^{[32]}$ 的偶联反应, 这些反应均 具有良好的底物兼容性并且反应条件温和, 实现了以不 同酰基源化合物为原料构建酮类化合物(Scheme 10).

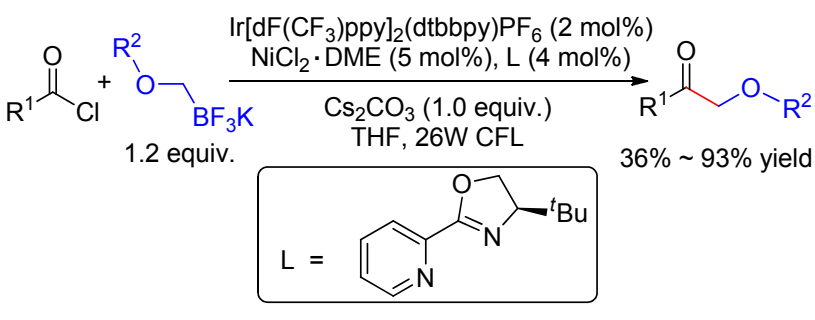

$$
\text { 1.5 equiv. }
$$

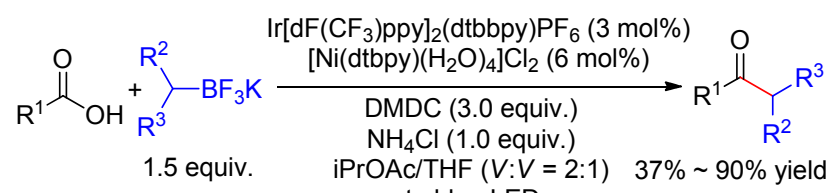$$
\text { r.t., blue LEDs }
$$

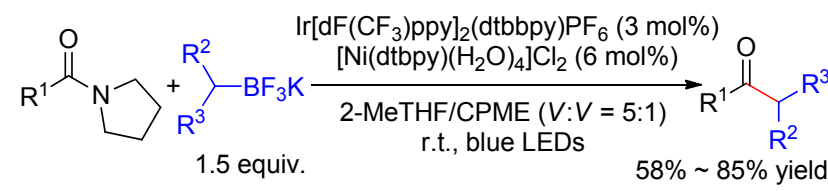

图式 10 光镍协同催化的烷基三氟嗍酸钾的酰基化反应

Scheme 10 Dual photoredox/nickel catalyzed acylation of alkyltrifluoroborates

2017 年, Doyle 小组和 Rovis 课题组 ${ }^{[33]}$ 合作报道了 光镍协同催化内消旋酸酐和茮基三氟嗍酸盐发生对映 选择性去对称化反应，制备了带有两个相邻手性中心的
酮酸化合物(Scheme 11). 该反应对茮基三氟碀酸钾兼容 性好，但是对于酸酐兼容性较差，当酸酐的 $\beta$ 位有取代 基时，产率和选择性出现明显的降低. 最后，作者通过 差向异构化实验发现催化剂与苄基三氟硼酸钾的相对 量对反应立体选择性有着明显的影响.
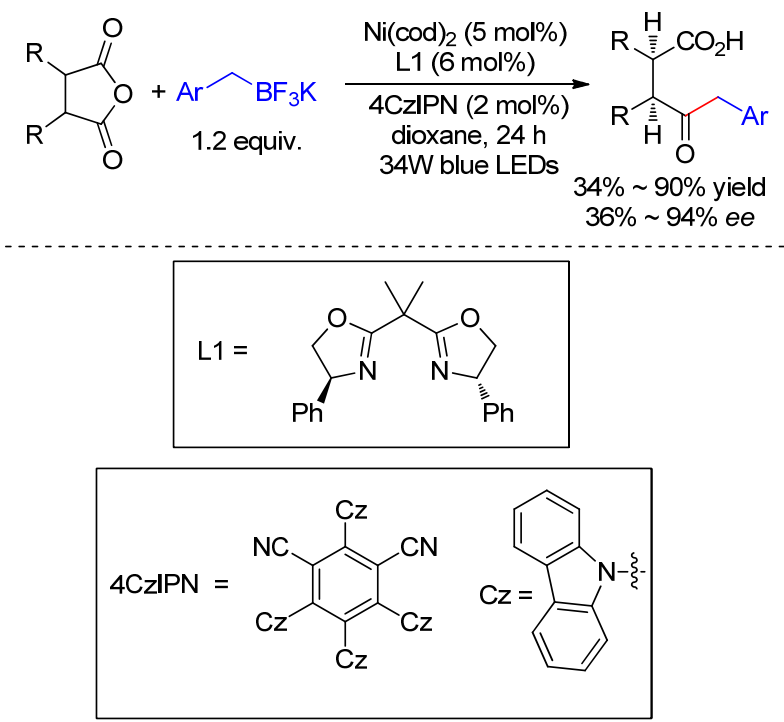

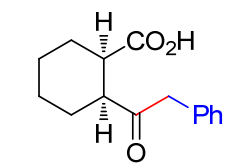

$77 \%$ yield $91 \%$ ee, $>20: 1 d r$<smiles>O=C(O)[C@H]1C[C@H]1C(=O)Cc1ccccc1</smiles>

$51 \%$ yield $51 \%$ ee, $19: 1 d r$<smiles>O=C(O)[C@H]1CCC[C@@H]1C(=O)Cc1ccccc1</smiles>

$84 \%$ yield $70 \%$ ee, > 20:1 $d r$<smiles>CC1CC[C@@H](C)[C@H](C(=O)O)[C]1C(=O)Cc1ccccc1</smiles>

$36 \%$ ee $9: 1 d r$

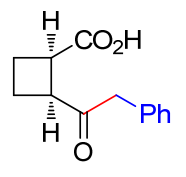

$70 \%$ yield $76 \%$ ee, $>20: 1 d r$<smiles>CC(C(=O)O)C(C)C(=O)Cc1ccccc1</smiles>

$70 \%$ yield $88 \%$ ee, $13: 1 d r$
图式 11 光镍协同催化内消旋酸酐的对映选择性去对称化反 应

Scheme 11 Dual nickel-photoredox-catalyzed enantioselective desymmetrization of cyclic meso-anhydrides

2018 年, 李朝军课题组 ${ }^{[34]}$ 发展了一种新颖的方法 实现了烷基以及芳基三氟硼酸钾的甲酰化反应(Scheme 12). 在该体系下，2,3-丁二酮不仅作为底物(烷基或者芳 基三氟嗍酸钾)的活化试剂，同时还作为酰基源参与反 应. 该反应条件温和，不需要光催化剂，同时使用水作 为溶剂, 具有绿色环境友好的特点. 作者推测反应机理 可能为: 烷基(芳基)三氟硣酸钾在溶液中部分以二氟烷 基(芳基)硼烷的形成存在，其可以和光活化的 2,3-丁二 酮氧自由基发生 $\mathrm{S}_{\mathrm{H}}$ 2(双分子均裂取代机理)过程释放出 烷基自由基.

2015 年, 陈以昀课题组 ${ }^{[35]}$ 在可见光催化下, 以 $\mathrm{Ru}$ 

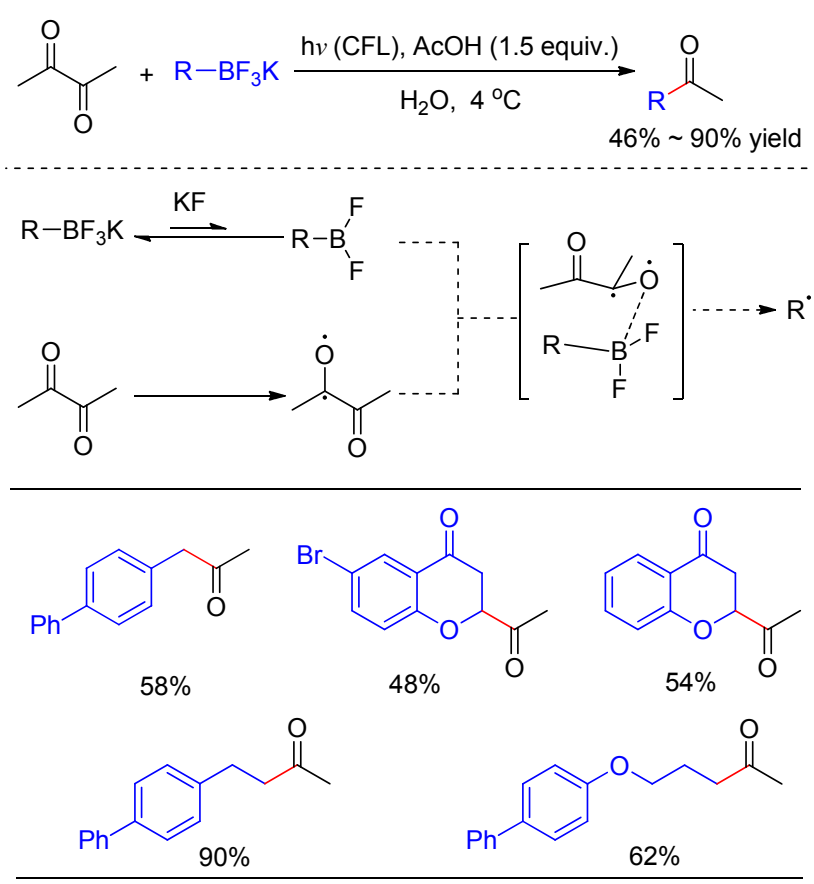

图式 12 2,3-丁二酮光共活化烷基三氟嗍酸钾

Scheme 12 Dual diacetyl/photoredox catalyzed activation of organotrifluoroborate

络合物为催化剂, 以 $\mathrm{BI}-\mathrm{OAc}$ 为氧化剂, 实现了烷基三 氟硼酸钾和 $\alpha, \beta$-不饱和羧酸的偶联反应(Scheme 13, a). 该反应底物适用范围广，官能团兼容性好，且高选择性 地得到反式产物. 通过对反应机理的研究他们发现该反 应的关键中间体为 $\alpha, \beta$-不饱和羧酸 $\mathrm{RCH}=\mathrm{CHCOOH}$ 与 $\mathrm{BI}-\mathrm{OAc}$ 生成的产物 $\mathrm{BI}-\mathrm{OOCCH}=\mathrm{CHR}$, 该中间体捕获 体系中烷基硼化合物生成的烷基自由基, 随后消除得到 产物并释放出二氧化碳气体以及 BI 自由基. 2016 年, Molander 课题组 ${ }^{[12]}$ 发展了非过渡金属光催化剂 $\mathrm{Na}_{2}-$ Eosin $\mathrm{Y}$ 催化的烷基三氟硼酸钾的烯基化反应(Scheme 13, b). 在该体系下, 不需要添加氧化剂, 以芳香烯基砜 作为自由基受体. 该反应同样具有较好的底物兼容性以 及高的立体选择性.

(a)

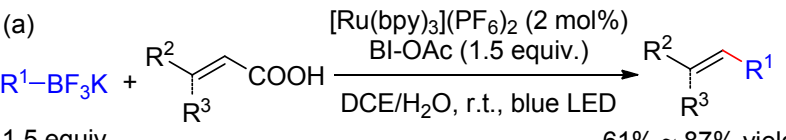

1.5 equiv.

$61 \% \sim 87 \%$ yield

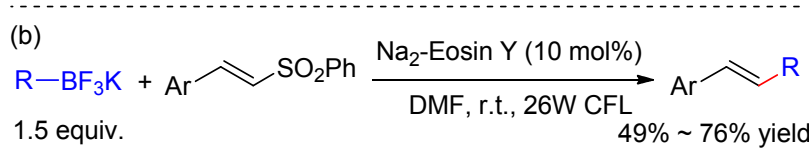

图式 13 光催化烷基三氟硓酸钾烯基化反应

Scheme 13 Photoredox catalyzed alkenylation of alkyltrifluoroborates

\section{$1.3 \mathrm{C}\left(\mathrm{sp}^{3}\right)-\mathrm{C}\left(\mathrm{sp}^{3}\right)$ 成键反应}

2013 年, Akita 课题组 ${ }^{[36]}$ 以 Ir 作为光催化剂, 实现了 $\alpha$ 烷氧基三氟硼酸钾脱硼构建 $\mathrm{C}\left(\mathrm{sp}^{3}\right)-\mathrm{C}\left(\mathrm{sp}^{3}\right)$ 键反应 (Scheme 14, a). 该反应经历 $\alpha$-烷氧基三氟硼酸钾的单电 子氧化生成烷氧基自由基, 并被贫电子烯烃捕获生成新 的自由基，随后被还原为碳负离子并质子解生成对应的 目标产物. 值得一提的是, 该反应在太阳光下也能很好 地进行. 随后, 基于此工作, 他们进一步发展了光催化 Boc 保护 $\alpha$-氨基三氟硼酸钾 ${ }^{[37]}$ 的烷基化反应，并合成了 具有生物活性的 $\gamma$-氨基丁酸(GABA)衍生物(Scheme 14, b). 在此工作基础上， $\alpha$-硫醚三氟硼酸钾 ${ }^{[38]}$ 也被成功地 应用于该策略(Scheme 14, c). 2015 年, 他们小组 ${ }^{[39]}$ 对该 反应进一步改进, 以廉价易得的常见染料 $\mathrm{MesAcr}^{+}$为光 催化剂, 实现了普通烷基三氟硼酸钾的烷基化反应 (Scheme 14, d). 此外, Yoshimi 课题组 ${ }^{[40]}$ 报道了菲作为 光催化剂, 对苯二腈作为共催化剂实现了芳基硼酸类化 合物与丙烯腈反应构建烷基腈化合物. 他们发现环已基 嗍酸也可在该条件下转化为对应的烷基腈化合物.

$$
\begin{aligned}
& \text { (a) } \quad \operatorname{Ir}\left(\mathrm{dFCF}_{3} \text { ppy }\right)_{2}(\text { bpy }) \mathrm{PF}_{6}
\end{aligned}
$$

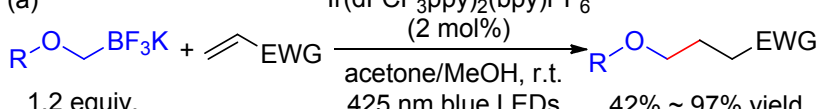

$$
\begin{aligned}
& \text {. }
\end{aligned}
$$

(b)

$$
\text { EWG } \frac{\operatorname{lr}\left(\mathrm{dFCF}_{3} \mathrm{ppy}\right)_{2}(\mathrm{bpy}) \mathrm{PF}_{6}(2 \mathrm{~mol} \%)}{\begin{array}{l}
\text { acetone/MeOH, r.t. } \\
425 \mathrm{~nm} \text { blue LEDs }
\end{array}}
$$

(c) $\quad \operatorname{Ir}\left(\mathrm{dFCF}_{3} \mathrm{ppy}_{2}(\mathrm{bpy}) \mathrm{PF}_{6}\right.$

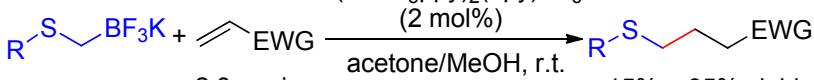

$$
\begin{aligned}
& 2.0 \text { equiv. } \quad 425 \mathrm{~nm} \text { blue LEDs } \quad 15 \% \sim 85 \% \text { yield }
\end{aligned}
$$

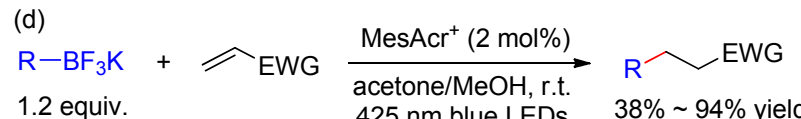

图式 14 贫电子烯烃作为受体的烷基硼化合物光催化转化

Scheme 14 Photoredox-catalyzed transformation of alkyl boron compounds using electron-deficient alkenes as accepter

2017 年, Molander 课题组 ${ }^{[41]}$ 以 $\alpha$-三氟甲基芳基乙烯 类化合物为自由基受体，以烷基三氟硼酸钾或者烷基硅 试剂作为自由基源, 实现了 1,1-二氟烯烃类化合物的构 建. 该方法具有优异的底物普适性，可兼容酯基，一级、 二级以及三级胺，醇羟基，醛基，炔基等多种官能团. 该策略为合成二氟乙烯类化合物提供了一种强有力的 方法(Scheme 15).

Meggers 课题组 ${ }^{[42]}$ 报道了在光照条件下，以手性八 面体 $\mathrm{Rh}$ 络合物和 $\mathrm{MesAcr}^{+}$作为催化剂, 烷基三氟硼酸 钾为自由基前体, 含吡唑或者咪唑导向基团的 $\alpha, \beta$-不饱 


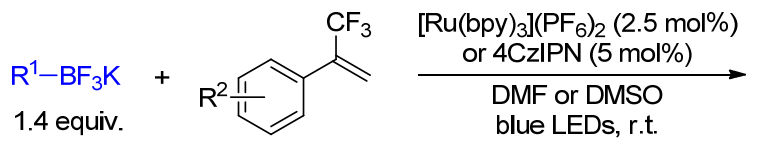

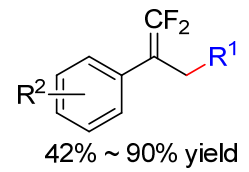

图式 15 光催化 1,1-二氟烯烃化合物的构建

Scheme 15 Photoredox construction of 1,1-difluoroalkene

和酮作为自由基受体, 通过自由基加成随后质子解得到 目标产物，实现了含导向基团的贫电子烯烃的手性烷基 化加成反应(Scheme 16). 该反应具有良好的底物兼容 性, 可兼容一级、二级以及三级烷基硼化合物, 值得一
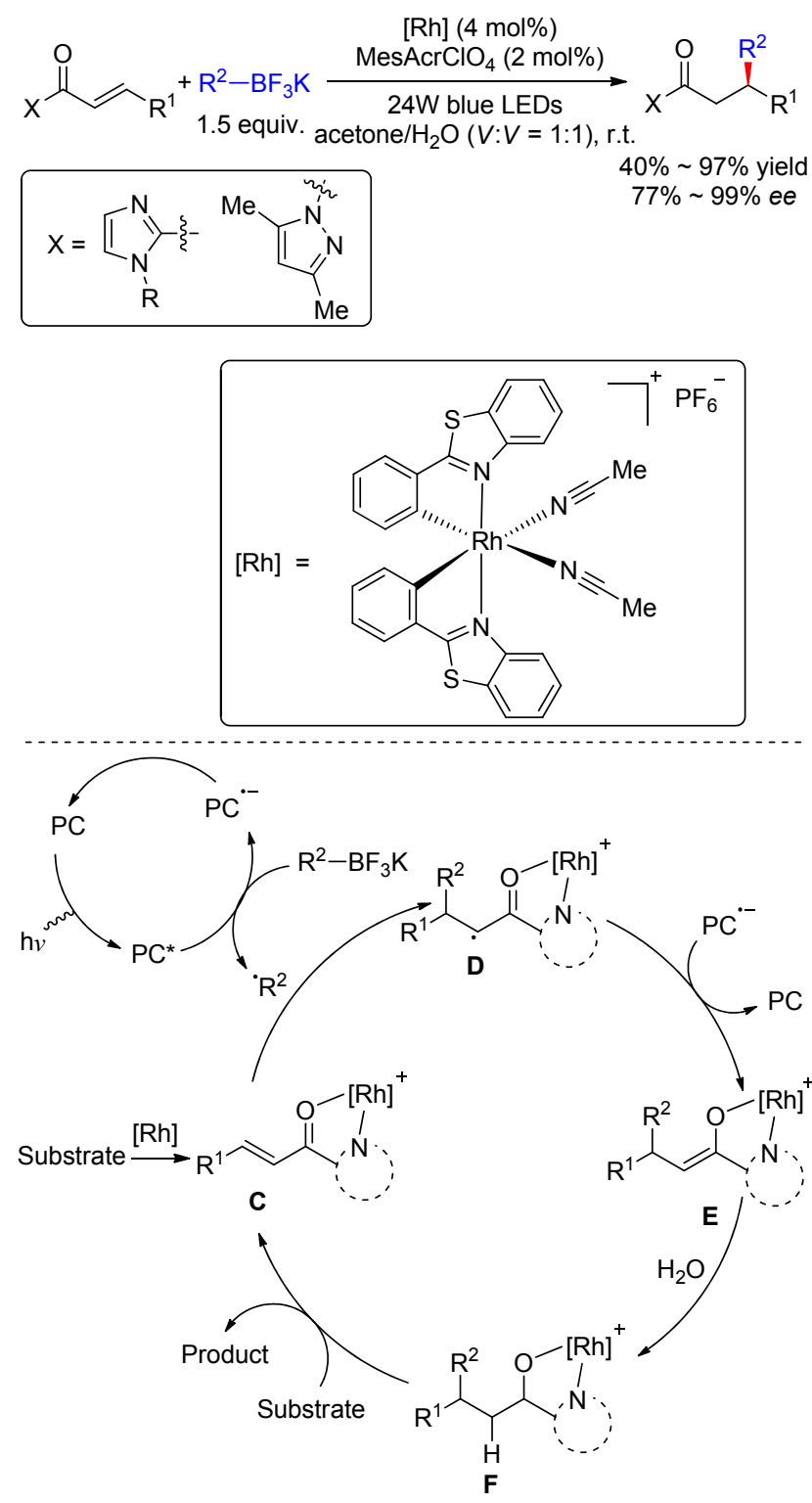

图式 16 光手性铑共催化烷基三氟硼酸钾对烯烃的手性加成 Scheme 16 Dual chiral rhodium complex/photoredox catalyzed enantioselective addition of alkyltrifluoroborates to alkenes
提的是，该反应具有优异的立体选择性. 随后他们和 Wiest 课题组 ${ }^{[43]}$ 合作通过 DFT 计算发现反应手性控制的 关键在于 $\alpha, \beta$-不饱和 2-酰基咪唑和手性 $\mathrm{Rh}$ 催化剂迅速 形成配合物 $\mathbf{C}, \mathbf{C}$ 作为体系中的烷基自由基捕获剂. 该 反应机理为: $\alpha, \beta$-不饱和 2-酰基咪唑和手性 $\mathrm{Rh}$ 催化剂迅 速形成配合物 $\mathbf{C}, \mathbf{C}$ 捕获体系中光生烷基自由基得到含 $\mathrm{Rh}$ 配合物自由基 $\mathbf{D}, \mathbf{D}$ 进一步被体系中还原态光催化剂 淬灭得到烯醇 $\mathrm{Rh}$ 化合物 $\mathbf{E}, \mathbf{E}$ 与水反应得到目标化合物 中间体 $\mathbf{F}$, 随后 $\mathbf{F}$ 与 $\alpha, \beta$-不饱和 2-酰基咪唑发生配体交 换得到目标产物以及参与反应的前体 $\mathbf{C}$.

2018 年, Hanna 课题组 ${ }^{[44]}$ 实现了温和条件下光催化 以烷基三氟硼酸钾为烷基化试剂的 $N$-芐叉苯胺烷基化. 该反应主要为烷基自由基对苄亚胺的加成(Scheme 17, a). 2019 年, Molander 课题组 ${ }^{[45]}$ 报道了温和条件下光催 化烷基三氟硼酸钾的 Petasis 反应(Scheme 17, b). 该反 应主要通过烷基自由基与原位生成的苄亚胺反应来实 现转化的. 此外, 该反应具有良好的官能团兼容性，可 以实现药物分子消炎疼、非诺贝特以及磺胺二甲氧嘧啶 的衍生化. 不仅如此，具有潜在胰高血糖素受体调节作 用药物的关键中间体也能用该方法合成.

(a)

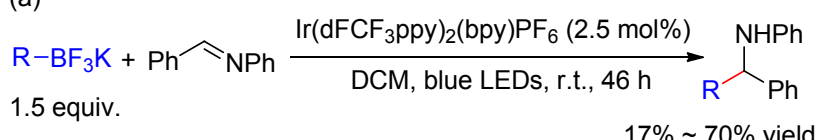

(b)
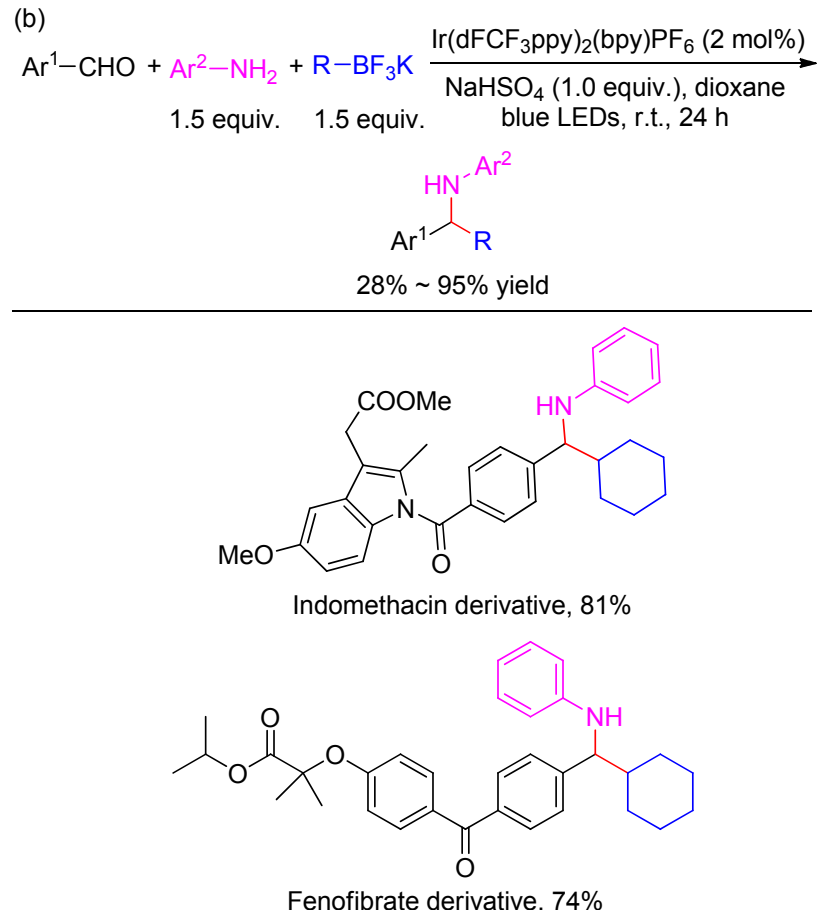

图式 17 光催化烷基三氟嗍酸钾对亚胺的加成反应

Scheme 17 Photoredox-catalyzed alkyltrifluoroborates addition to imines

2018 年, 肖文精课题组 ${ }^{[46]}$ 实现了光镍协同催化的 
苠基氮杂环丙烷的开环合成 $\beta$-取代胺化合物 (Scheme 18). 在该体系中, 以茮基三氟嗍酸钾作为自由基源, 选 择性地加成在苄基氮杂环丙烷的苄位. 然而, 该反应底 物存在一定的限制性, 脂肪烷基三氟硼酸钾无法发生该 转化.

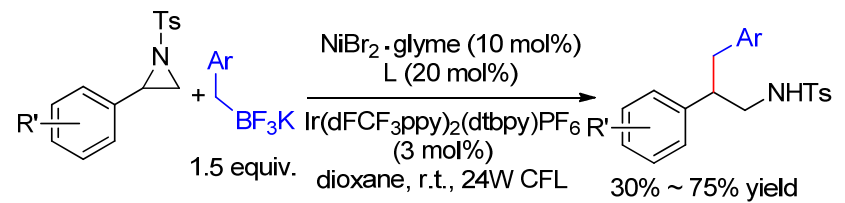

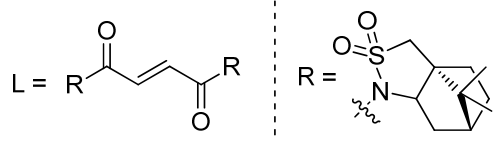

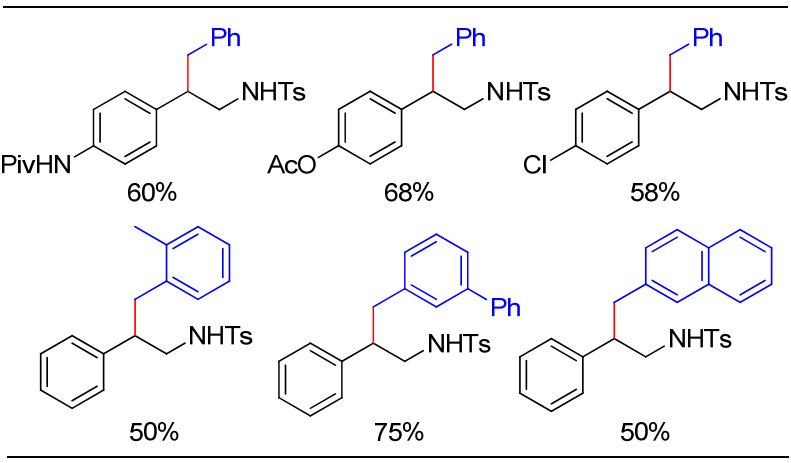

图式 18 光镍协同催化 $\beta$ 取代胺的合成 Scheme 18 Dual photoredox/nickel-catalyzed synthesis of $\beta$ substitued amines

2018 年, 陈以昀课题组 ${ }^{[47]}$ 报道了光催化合成 $\alpha$-季 碳中心环酮以及呋喃酮类化合物. 该反应以烷基喼酸作 为自由基源. 反应的关键在于烯丙醇与环碘试剂 (BI-OAc)形成配合物活化烯丙醇(Scheme 19).

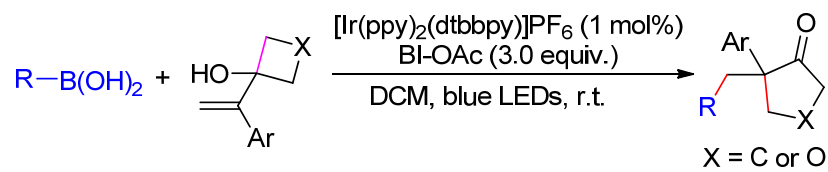

图式 19 光催化烷基嗍酸与烯丙醇的加成重排反应

Scheme 19 Photoredox the addition/rearrangement reaction of alkyl boric acid with allyl alcohols

2019 年, 陈以昀课题组 ${ }^{[48]}$ 报道了无需光催化剂同 时无需添加剂的光催化烷基嗍化合物(嗍酸或四氟硼酸 钾)对 $\alpha$-酮酸的自由基加成反应(Scheme 20). 该反应可 以在有机溶剂与水混合溶剂中进行, 并成功避免了 $\alpha$-酮 酸自身发生自由基脱羧, 也避免产物发生进一步的碳一 碳键断裂. 通过对反应机理的研究, 他们发现 $\alpha$-酮酸与 烷基硼酸可以形成全新的分子复合物. 该复合物具有可 见光吸收并且在可见光照射下，可发生脱嗍生成烷基自 由基. 此外, 该复合物还可促进烷基自由基对酮酸的加
成并抑制产物碳一碳键断裂，另外，使用流动光反应装 置可以合成克级规模的乳酸类药物分子关键中间体. 据 此，他们实现了以砋试剂如硼酸三甲酯作为 Lewis 酸催 化烷基二氢吡啶衍生物与 $\alpha$-酮酸的自由基加成反应.
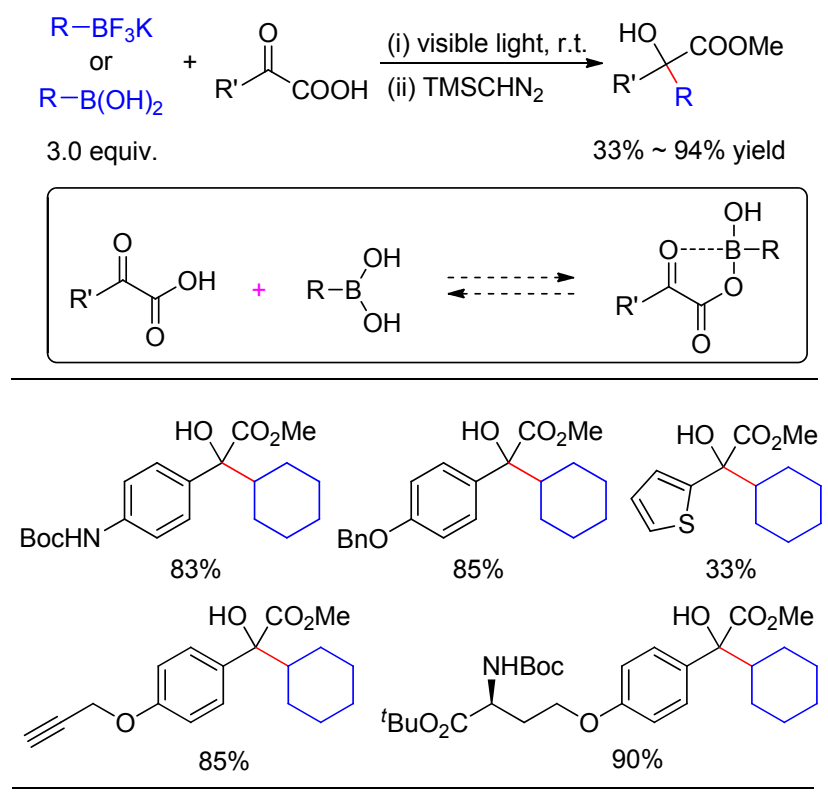

图式 20 光催化烷基嗍化合物对 $\alpha$-酮酸的加成反应 Scheme 20 Photoredox-catalyzed alkyl boron compounds addition to ketoacids

2019 年, 韩满意与王敏课题组 ${ }^{[49]}$ 以烷基硼酸为原 料, 采用 BI-OAc 作为活化试剂, 以丙烯酰胺为自由基 捕获剂，构建了 3,3-二取代吲哚酮结构(Scheme 21). 该 反应具有条件温和及不需要金属催化剂的特点. 此外, 在该体系下烯基硼酸也能转化为相应的产物，但是芳基

$$
\text { 2.0 equiv. }
$$<smiles>CN1C(=O)C(C)(CC2CCC2)c2ccccc21</smiles><smiles>CN1C(=O)C(C)(CC2CCCCC2)c2cccnc21</smiles><smiles>CN1C(=O)c2ccccc2C(CC2CCCCC2)(CC2CCCCC2)C1=O</smiles>

$51 \%$ $68 \%$

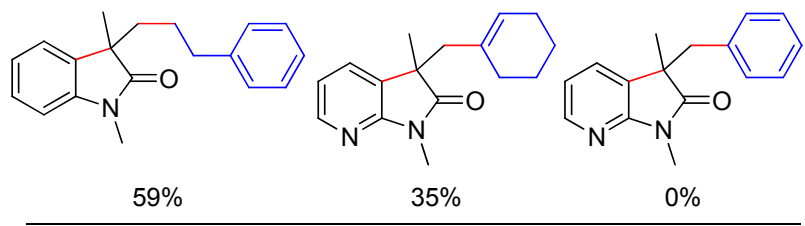

图式 21 光催化丙烯酰胺的烷基化反应

Scheme 21 Photoredox-catalyzed alkylation of acrylamides 
嘲无法实现该转化.

目前光催化的自由基反应多数实现了二组分反应, 实现三组分参与的反应更具有挑战性. 2019 年, Molander 课题组 ${ }^{[50]}$ 报道了有机染料光催化的脱氢丙氨酸烷 基化氟化反应，合成多种 $\alpha$-氟取代氨基酸衍生物 (Scheme 22). 该反应可兼容炔基、酮羰基、酰胺基团、 酯基以及磺酰胺基团等多种官能团. 该反应的机理为光 催化烷基三氟硼酸钾生成烷基自由基，随后被脱氢丙氨 酸捕捉并被 Selectfluor 淬灭得到目标产物.
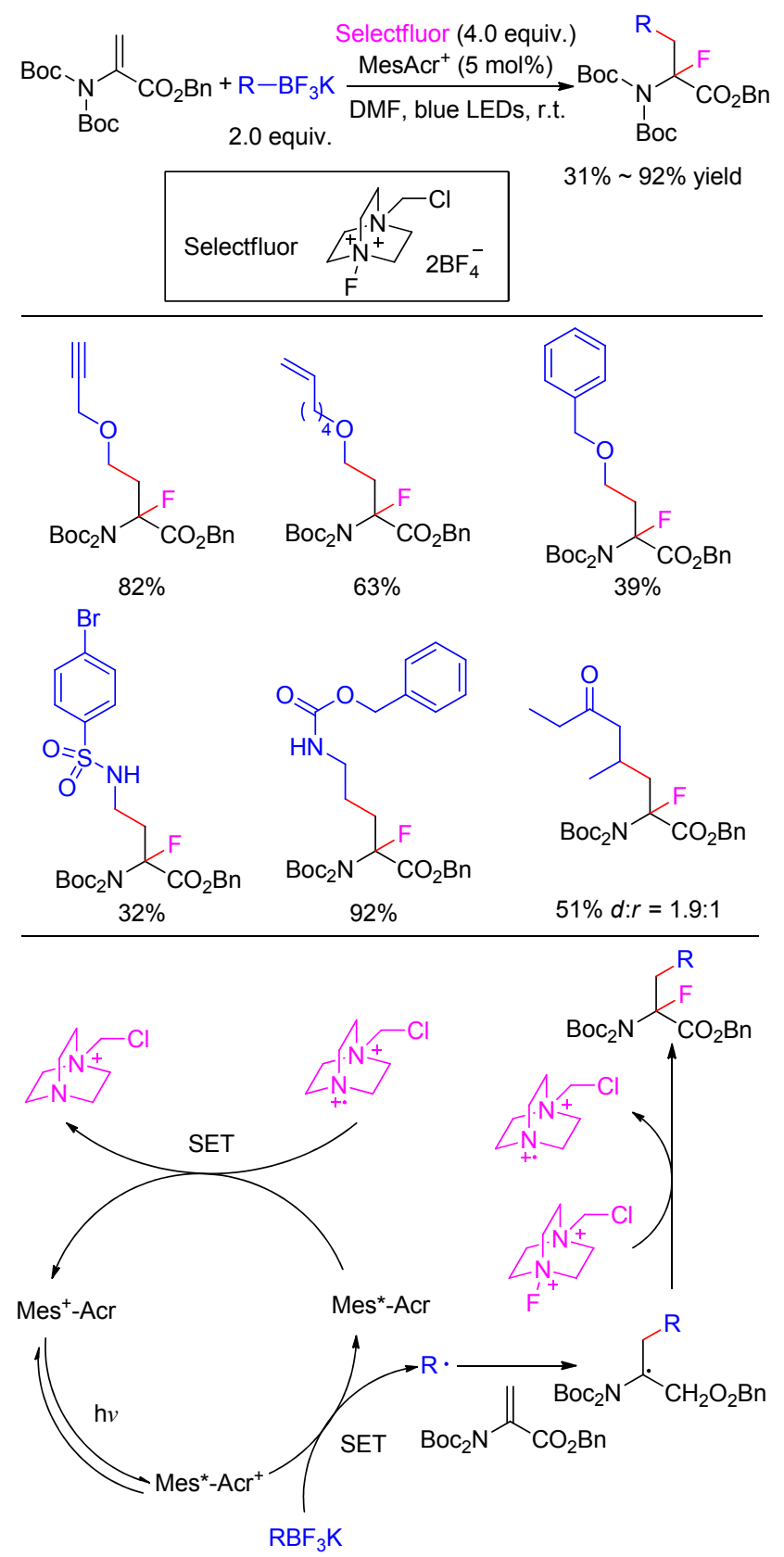

图式 22 光催化脱氢丙氨酸烷基化氟化反应

Scheme 22 Photoredox-catalyzed carbofluorination of dehydroalanine

2019 年, Molander 课题组 ${ }^{[51]}$ 报道了光镍协同催化的
芳基溴、烷基三氟嗍酸钾以及乙烯基频哪醇硼酸酯的三 组分反应合成烷基硼化合物(Scheme 23). 该反应具有 条件温和及底物普适性好的特点. 该反应的机理为: 光 催化烷基三氟硼酸钾生成烷基自由基，随后烷基自由基 被烯基硼捕获生成 $\alpha$-嗍酸酯自由基，该自由基被 $\mathrm{Ni}(0)$ 捕获生成 $\mathrm{Ni}$ (I)中间体并与芳基溴氧化加成生成 $\mathrm{Ni}(\mathrm{III})$ 化合物, Ni(III)化合物进一步发生还原消除得到目标产 物. 基于该策略，他们以 $71 \%$ 的收率制备革兰氏阳性细 菌胸苷酸激酶抑制剂(TK-666)的重要中间体.

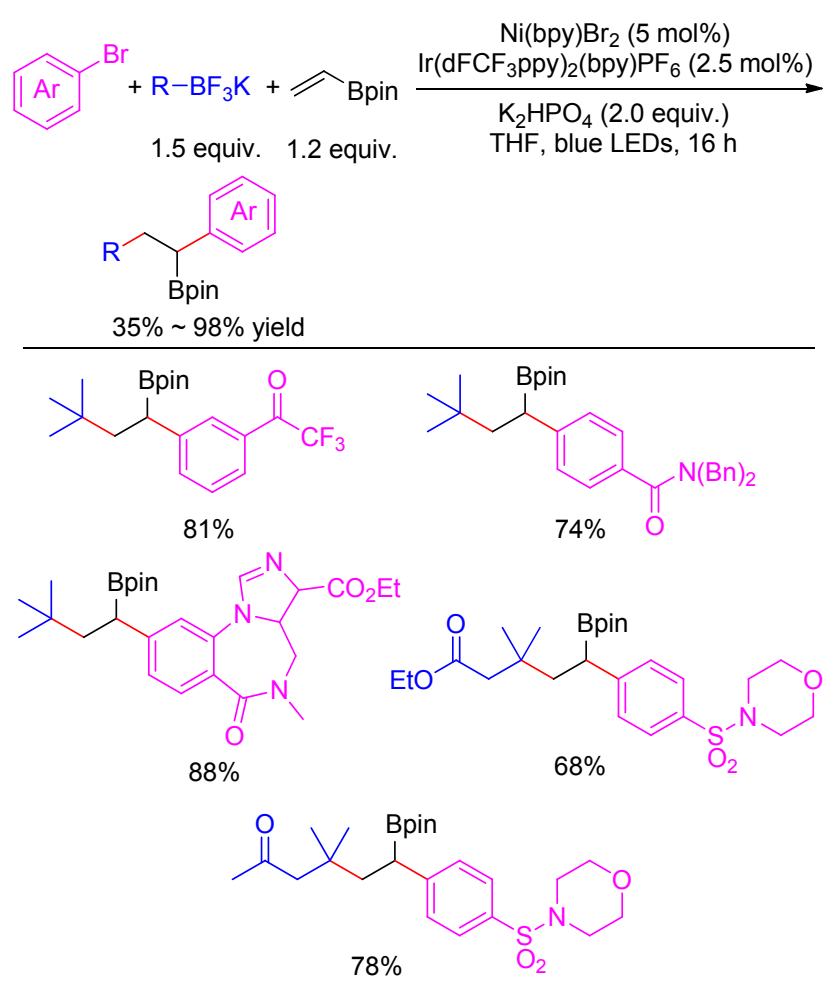

图式 23 光催化烷基嗍频哪醇嗍酸酯的合成

Scheme 23 Photoredox catalyzed synthesis of alkyl pinacol boronates

2017 年, Ley 课题组 ${ }^{[52]}$ 基于烷基硼酸酯以及烷基硼 酸的 Lewis 酸性, 采用催化量的 Lewis 碱作为活化试剂, 在光照条件下使用 Ir 催化剂催化其生成烷基自由基. 以 贫电子烯烃这一常见自由基受体为捕获剂, 实现了 $\mathrm{C}\left(\mathrm{sp}^{3}\right)-\mathrm{C}\left(\mathrm{sp}^{3}\right)$ 键的构建(Scheme 24, a). 作者发现常见 的 Lewis 碱如 DMAP、奎宁环、3-羟基奎宁环以及三苯 基膦均可实现烷基硼酸酯的活化. 在该催化体系下，烷 基硼酸底物兼容性较烷基硼酸酯好. 2018 年，他们小 组 ${ }^{[53]}$ 对反应进行了优化, 采用 Mes-Acr-4 作为光催化剂, 通过流动反应装置，提高了反应效率。基于此，他们通 过两步合成四种中枢神经系统 GABA 家族药物. 随后 2019 年, 他们 ${ }^{[54]}$ 继续以该策略实现了茮基频哪醇硼酸 酯与芳香醛酮的加成反应，高效地合成了二级以及三级 
醇类化合物(Scheme 24, b).

(a)

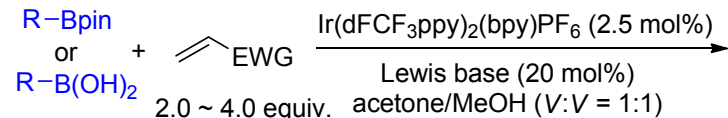

$$
\begin{aligned}
& \text { R } \text { EWG } \\
& 32 \% \sim 91 \% \text { yield }
\end{aligned}
$$

$\underbrace{N_{77 \%}^{N}}_{75 \%}$

(b)

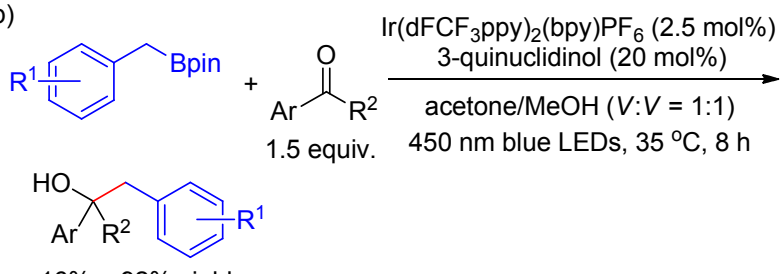

$16 \% \sim 92 \%$ yield

图式 24 Lewis 碱活化烷基嗍化合物的光催化转化

Scheme 24 Photeredox conversion of alkyl boron compounds activated by Lewis base

2018 年, 许孝良课题组 ${ }^{[55]}$ 实现了光催化烷基三氟 硣酸酯与 Baylis-Hillman 反应产物衍生物的偶联反应, 合成了一系列 $\alpha, \beta$-不饱和羧酸酯衍生物. 该反应具有良 好的底物兼容性. 遗憾的是, 该反应产物的立体 $(E / Z)$ 选 择性稍差. 随后 2019 年, 他们小组 ${ }^{[56]}$ 以烷基硼酸酯或 者烷基硼酸作为自由基源实现了类似的转化. 在该体系 下, 催化量的碱 1,4-二氮杂二环[2.2.2]辛烷(DABCO)作 为三配位硼的活化试剂(Scheme 25).
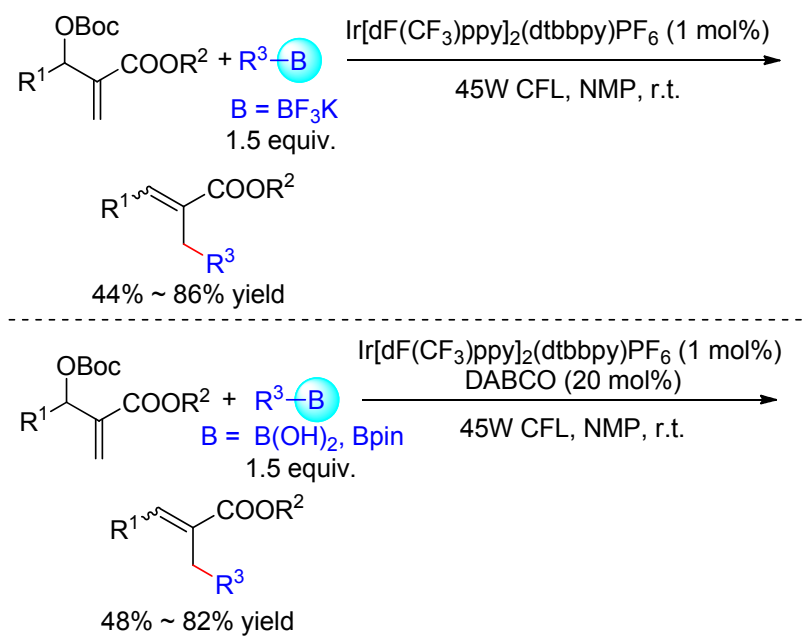

图式 25 光催化 $\alpha, \beta$-不饱和羧酸酯的合成

Scheme 25 Photoredox catalyzed synthesis of $\alpha, \beta$-unsaturated carboxylic esters

2019 年, Aggarwal 课题组 ${ }^{[57]}$ 报道了烷基频哪醇硼酸 酯脱硼环丁烷化反应(Scheme 26). 作者发现相比于烷
基三氟砋酸钾，苯基锂与烷基硼酸酯形成的四配位硼具 有更低的氧化电势，可以被廉价易得的有机染料 $4 \mathrm{CzIPN}$ 单电子氧化生成烷基自由基，同时伴随着苯基 频哪醇硼酸酯副产物的生成. 基于此，当以 $\gamma$-碘代烯酸 酯作为自由基捕获剂时，通过自由基加成以及脱碘环化 反应得到目标环丁烷类化合物. 此外，当加入不同碳数 的末端卤代烯酸酯，三环、五环、六环以及七环均可被 构建. 与 Ley 课题组报道方法相比，该方法具有更加良 好的底物兼容性，可以兼容多种一级、二级以及三级烷 基频哪醇硼酸酯，并且多种药物以及天然产物硼化衍生 物也可以以良好的收率得到目标产物.

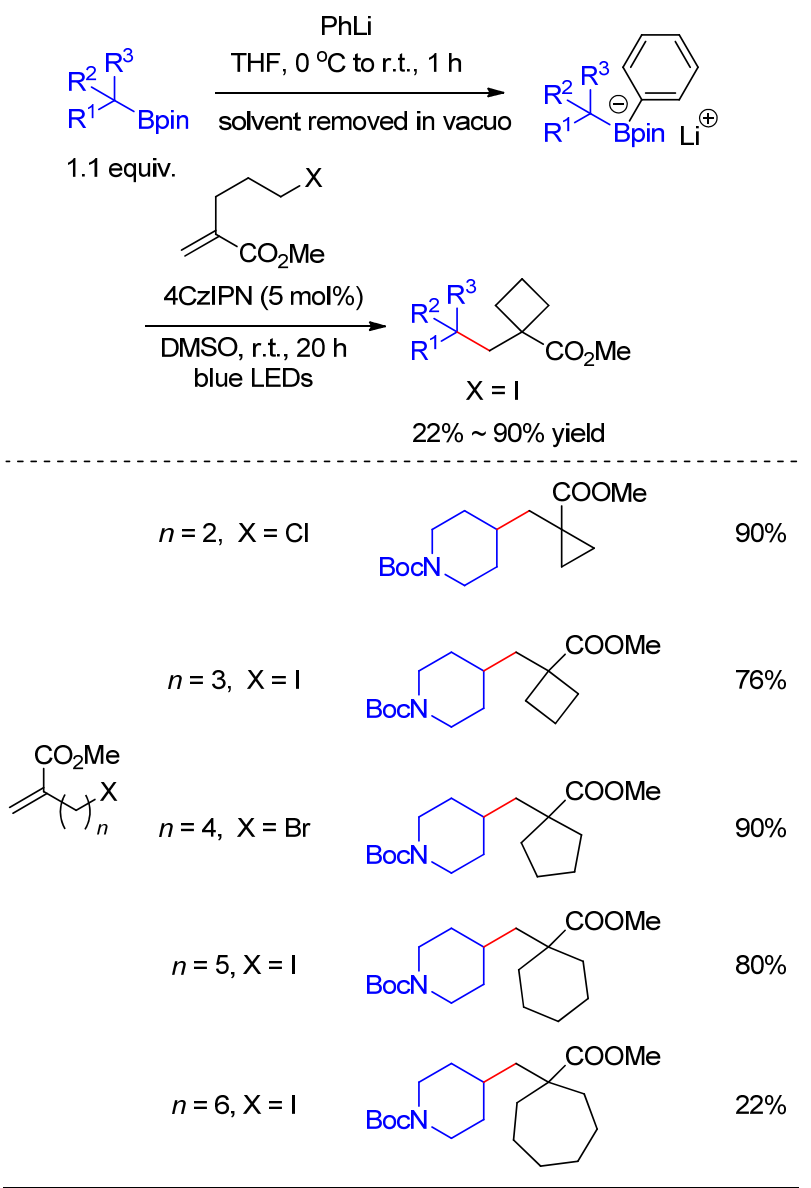

图式 26 光催化烷基频哪醇硼酸酯的环化反应 Scheme 26 Photoredox catalyzed cyclization of alkyl pinacol boronates

在此工作基础上, Aggarwal 课题组 ${ }^{[58]}$ 报道了 1,2-二 硼酸酯的选择性烷基化反应(Scheme 27). 他们发现在 光催化 1,2-双硼酸酯的脱嗍化反应中，等化学计量的活 化试剂芳基锂优先同位阻小的嘲形成四配位硼，随后发 生光催化脱硣过程生成 $\beta$-砋酸酯烷基自由基，该自由基 迅速经历 1,2-嗍转移过程形成热力学稳定的多取代基大 位阻的烷基自由基，随后烷基自由基被贫电子烯烃捕 捉，从而允许空间受阻的嗍酸酯发生选择性转化. 该反 
应不需要金属催化剂并且底物能兼容大多数不同取代 基. 此外，作者通过一系列实验来验证该反应的机理， 当以 1,2,3-含伯嗍、仲嗍以及叔嗍化合物作为底物时, 热 力学控制有利于最稳定的叔碳自由基中心作为反应位 点. 当用三氟甲基亚磺酸钠生成三氟甲基自由基处理烯 丙基硼酸酯, 目标产物发生 1,2-硼迁移. DFT 计算也支 持了 1,2-迁移过程. 此外, 通过硼同位素实验证明了该 迁移过程并且证实形成四配位硼位点位于位阻小的砋 酸酯上.
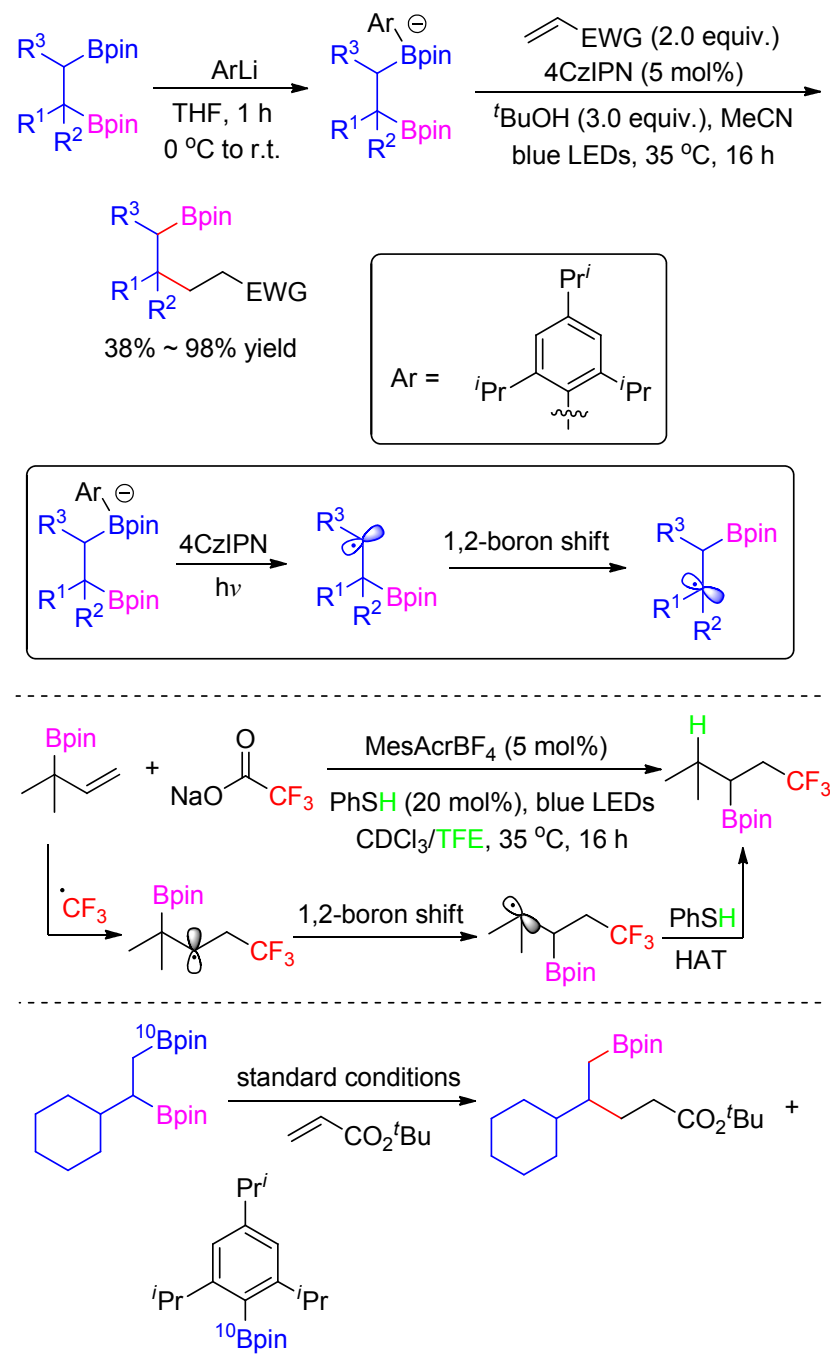

图式 27 光催化 1,2-二烷基嗍酸酯的选择性转化

Scheme 27 Photoredox catalyzed selectively alkylarylation of 1,2-bis-alkyl pinacol boronates

\section{$2 \mathrm{C}$ - $\mathrm{O}$ 成键反应}

目前，实现烷基嗍化合物脱硼构建 $\mathrm{C}-\mathrm{O}$ 键常用的 方法是过量氧化剂氧化, 如 $\mathrm{H}_{2} \mathrm{O}_{2} / \mathrm{NaOH} 、 \mathrm{NaBO}_{4}$ 等. 2012 年, Akita 课题组 ${ }^{[59]}$ 报道了第一例光催化烷基硼酸 盐的脱硼 $\mathrm{C}-\mathrm{O}$ 成键反应(Scheme 28). 该反应机理为: 激发态的 $\mathrm{Ir}$ 光催化剂单电子氧化烷基硼酸盐化合物生
成烷基自由基，随后烷基自由基被四甲基哌啶氧化物 (TEMPO)捕获生成目标产物. 尽管该反应体系的底物具 有一定的局限性，如不能将简单的一级以及二级烷基三 氟硼酸盐类化合物转化为相应的目标产物, 但是该反应 的发现为烷基硼化合物的活化提供了一种新的策略，同 时也表明四配位嗍络合物可在光催化条件下作为一种 良好的自由基前体.
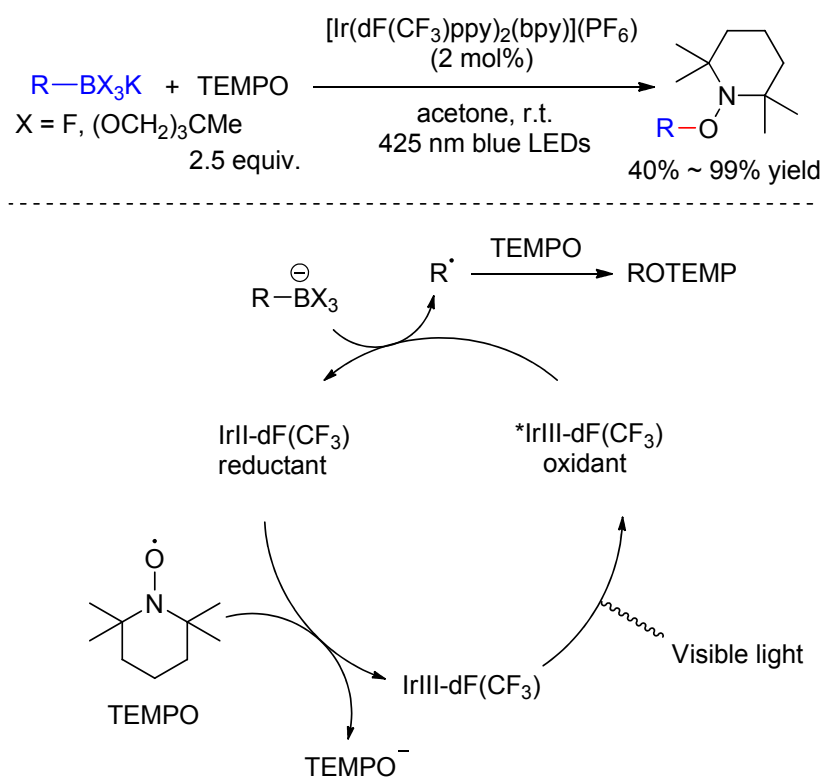

图式 28 光催化烷基嗍酸盐的碳氧成键反应

Scheme 28 Construction of $\mathrm{C}-\mathrm{O}$ bonds by Photoredox catalyzed alkylborates deboronation

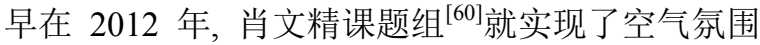
下，光催化氧化芳基硣酸生成酚的反应. 然而，烷基硼 化合物的相应转化却缺乏报道. 2018 年, 张博课题组 ${ }^{[61]}$ 以有机染料玫瑰红为光催化剂, 乙醇为溶剂, 在空气氛 围温和条件下将烷基以及芳基硼化合物氧化为对应的 醇或者酚(Scheme 29). 在该催化体系下, 空气中的氧气 作为氧化剂. 当以手性砋化合物为底物时，反应得到对 应的手性醇，表明该体系下，烷基硼化合物并没有形成 碳中心自由基. 机理研究表明，该体系可能是光催化原 位生成双氧水参与了有机硼的氧化过程.

\section{$3 \mathrm{C}$ - S 成键反应}

目前，通过光催化实现烷基硼化合物脱硼构建碳一 硫键的报道较少. 2018 年, 吴劼课题组 ${ }^{[62]}$ 首次报道了通 过光催化烷基三氟硼酸钾、 $\mathrm{DABCO} \cdot\left(\mathrm{SO}_{2}\right)_{2}$ 和贫电子取 代烯烃的三组分反应合成烷基磺酰基化合物的方法 (Scheme 30,a). 该反应可以兼容不同的烷基三氟嗍酸 钾，同时含有多种敏感官能团如羟基、酮羰基以及硝基 的贫电子烯烃在该反应条件下也可被兼容. 作者认为该 


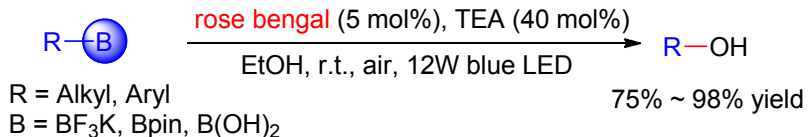

$\mathrm{B}=\mathrm{BF}_{3} \mathrm{~K}, \mathrm{Bpin}, \mathrm{B}(\mathrm{OH})_{2}$
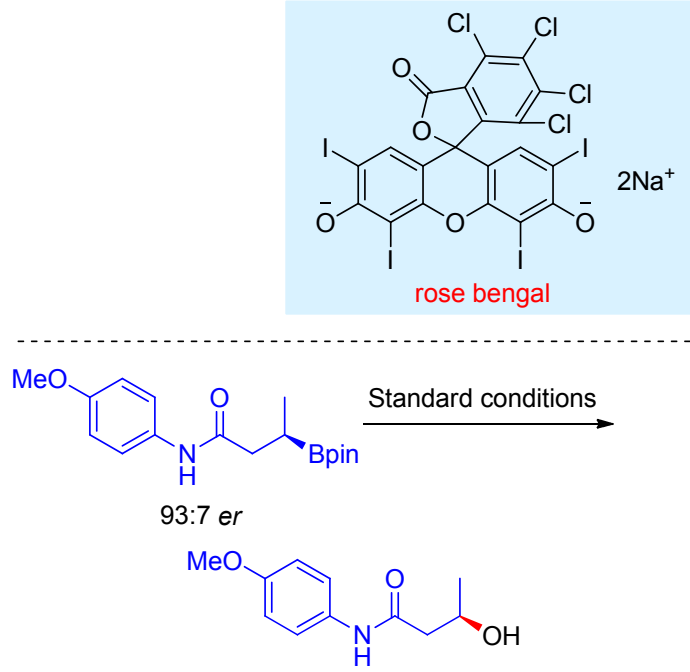

$86 \%$ yield, $93: 7$ er

图式 29 光催化空气氧化烷基硼化合物

Scheme 29 Photoredox catalyzed air oxidation of alkyl boron compounds

反应的机理为激发态光催化剂将烷基三氟硼酸钾氧化 为烷基自由基，随后烷基自由基捕获体系中二氧化硫生 成砜自由基, 进一步砜自由基与烯烃加成并氧化还原态 光催化剂生成对应的碳负离子, 最后质子化得到目标产 物. 基于此工作，他们 ${ }^{[63]}$ 又报道了类似条件下生成的砜 自由基与烯丙基溴反应得到烯丙基砜(Scheme 30, b). 此外, 该小组 ${ }^{[64]}$ 还报道了砜自由基与芳香末端炔烃反 应得到的烯基砜产物, 不同的是, 该反应需要 $\mathrm{Cu}(\mathrm{OTf})_{2}$ 作为共催化剂. 铜催化剂捕获砜自由基生成砜铜物种, 该物种与芳香末端炔烃加成生成烯基铜物种, 随后质子 化得到目标产物(Scheme 30, c). 2020 年, 该小组 ${ }^{[65]}$ 进一 步以烷基三氟硼酸钾作为自由基源, 并通过 $\mathrm{Na}_{2} \mathrm{~S}_{2} \mathrm{O}_{5}$ 捕 获生成对应的砜自由基, 最后与炔溴反应得到炔砜类化 合物(Scheme 30, d).

\section{4 脱䂽质子化反应}

烷基碳硼键脱硼质子化具有一定的研究价值. 2019 年, Studer 课题组 ${ }^{[66]}$ 报道了光催化的烷基硼酸酯脱硼氢 化反应(Scheme 31). 他们使用苯基锂作为亲核试剂将 烷基硼化合物预先制备为四配位硼络合物，随后以苯硫 酚作为氢源, Ir 配合物作为光催化剂实现了烷基频哪醇 硣酸酯的氢化反应. 当以烯烃为原料时, 结合硼氢化反 应以及 Matteson 同系化反应，实现了烯烃反马氏甲基化 反应. 此外, 他们进一步拓展了该反应的应用价值, 以 Boc 保护四氢吡咯为原料, 通过简单的四步转化合成了

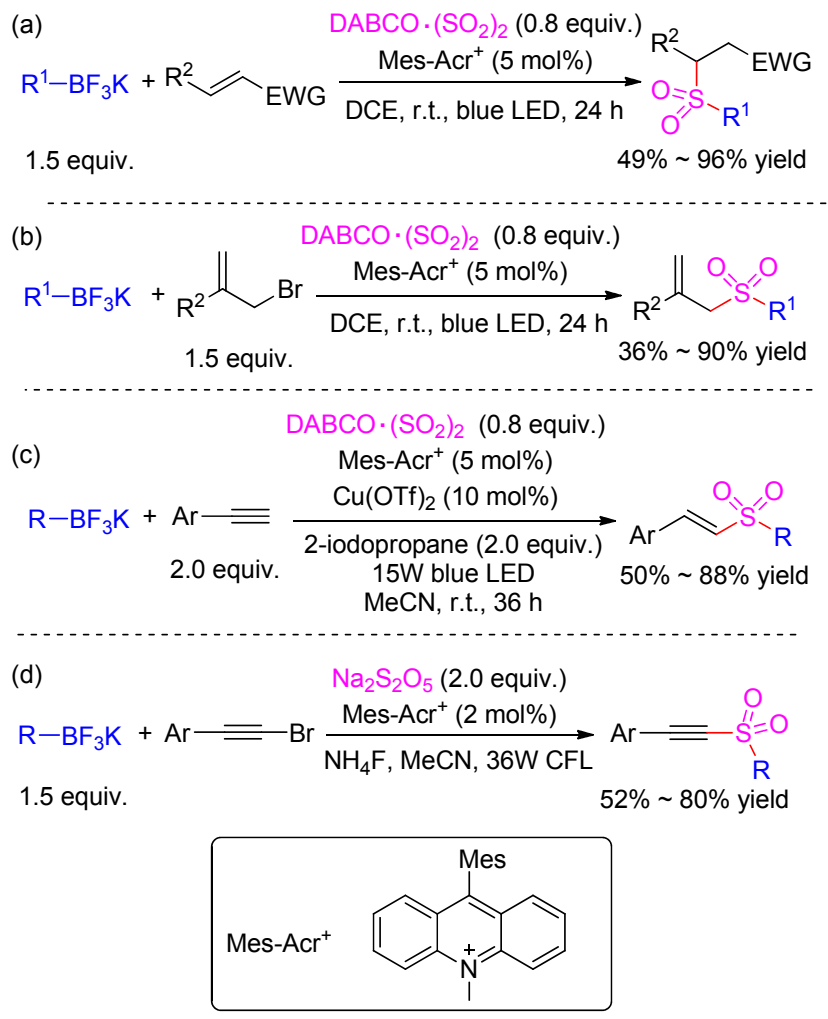

图式 30 光催化烷基三氟嗍酸钾构建 $\mathrm{C}-\mathrm{S}$ 键

Scheme 30 Construction of $\mathrm{C}-\mathrm{S}$ bonds by photoredox catalyzed alkyltrifluoroborates

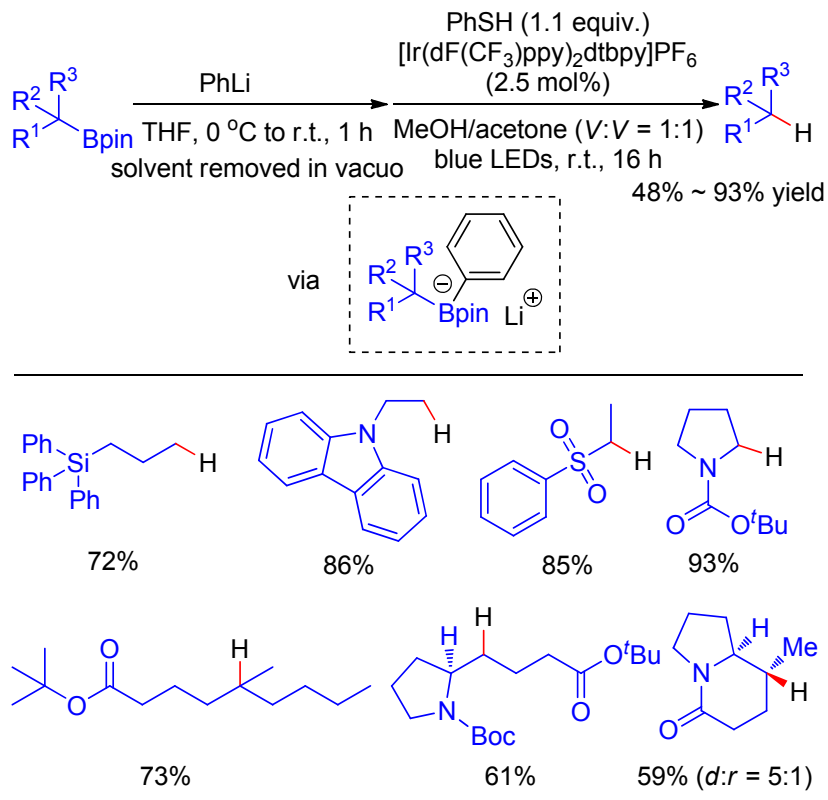

图式 31 光催化烷基嗍酸酯的氢化反应

Scheme 31 Photoredox catalyzed hydrogenation of alkyl boronates

吲哚里西啶 209B 中间体和可卡因关键中间体.

\section{5 总结与展望}

综上所述，近些年来通过光催化的手段活化烷基硼 
化合物极大地丰富了其转化类型, 并且反应具有条件温 和及官能团兼容性广的优点. 然而, 该领域依旧存在一 系列挑战需要解决. 首先, 目前烷基嗍化合物大多原料 为烷基三氟硼酸钾, 其合成需要通过其他硼化合物的进 一步转化制备. 因此, 以易制备易分离的烷基喼酸酯直 接作为自由基源具有重要的研究意义以及更好的经济 性. 仅有的几例烷基硼酸酯的活化存在着底物普适性较 差、活化方式单一的问题. 故而, 发展更为廉价、催化 量、普适性好烷基硼酸酯活化试剂显得十分有必要. 其 次，受制于自由基受体的种类，光催化烷基硼化合物脱 硓成键类型依旧十分有限，进一步拓展其成键类型具有 十分重要的意义. 最后, 目前关于该类反应的不对称催 化体系十分少见，如何有效控制反应的立体选择性仍然 是一个巨大的挑战.

\section{References}

[1] Miyaura, N.; Yamada, K.; Suzuki, A. Tetrahedron Lett. 1979, 20, 3437.

[2] Brown, H. C.; Tierney, P. A. J. Am. Chem. Soc. 1958, 80, 1552.

[3] (a) Chan, D. M. T.; Monaco, K. L.; Wang, R.-P.; Winters, M. P. Tetrahedron Lett. 1998, 39, 2933.

(b) Evans, D. A.; Katz, J. L.; West, T. R. Tetrahedron Lett. 1998, 39 , 2937.

(c) Lam, P. Y. S.; Clark, C. G.; Saubern, S.; Adams, J.; Winters, M. P.; Chan, D. M. T.; Combs, A. Tetrahedron Lett. 1998, 39, 2941.

[4] Zweifel, G.; Arzoumanian, H.; Whitney, C. C. J. Am. Chem. Soc. 1967, 89, 3652.

[5] Lennox, A. J.; Lloyd-Jones, G. C. Chem. Soc. Rev. 2014, 43, 412.

[6] (a) Leonori, D.; Aggarwal, V. K. Angew. Chem., Int. Ed. 2015, 54, 1082 .

(b) Sandford, C.; Aggarwal, V. K. Chem. Commun. 2017, 53, 5481.

[7] (a) Chen, Y.; Lu, L.-Q.; Yu, D.-G.; Zhu, C.-J.; Xiao, W.-J. Sci. China: Chem. 2018, 62, 24.

(b) Chen, D.; Liu, J.; Zhang, X.; Jiang, H.; Li, J. Chin. J. Org. Chem. 2019, 39, 3353 (in Chinese).

(陈丹, 刘剑沉，张馨元，蒋合众，李加洪，有机化学，2019，39, 3353.)

(c) Dai, X.; Xu, X.; Li, X. Chin. J. Org. Chem. 2013, 33, 2046 (in Chinese).

(戴小军，许孝良，李小年，有机化学, 2013, 33, 2046.)

[8] Duret, G.; Quinlan, R.; Bisseret, P.; Blanchard, N. Chem. Sci. 2015, 6,5366

[9] Duan, K.; Yan, X.; Liu, Y.; Li, Z. Adv. Synth. Catal. 2018, 360, 2781.

[10] Huang, H.; Zhang, G.; Gong, L.; Zhang, S.; Chen, Y. J. Am. Chem. Soc. 2014, 136, 2280.

[11] Dai, J. J.; Zhang, W. M.; Shu, Y. J.; Sun, Y. Y.; Xu, J.; Feng, Y. S.; $\mathrm{Xu}$, H. J. Chem. Commun. 2016, 52, 6793.

[12] Heitz, D. R.; Rizwan, K.; Molander, G. A. J. Org. Chem. 2016, 81, 7308.

[13] Shi, D.; Xia, C.; Liu, C. CCS Chem. 2020, 2, 1718.

[14] Tellis, J. C.; Primer, D. N.; Molander, G. A. Science 2014, 345, 433.

[15] Gutierrez, O.; Tellis, J. C.; Primer, D. N.; Molander, G. A.; Kozlowski, M. C. J. Am. Chem. Soc. 2015, 137, 4896.

[16] Primer, D. N.; Karakaya, I.; Tellis, J. C.; Molander, G. A. J. Am. Chem. Soc. 2015, 137, 2195.

[17] Primer, D. N.; Molander, G. A. J. Am. Chem. Soc. 2017, 139, 9847.

[18] Alam, R.; Molander, G. A. J. Org. Chem. 2017, 82, 13728.

[19] Ryu, D.; Primer, D. N.; Tellis, J. C.; Molander, G. A. Chem.-Eur. J. 2016, 22, 120 .
[20] Tellis, J. C.; Amani, J.; Molander, G. A. Org. Lett. 2016, 18, 2994.

[21] Matsui, J. K.; Molander, G. A. Org. Lett. 2017, 19, 436.

[22] Lima, F.; Kabeshov, M. A.; Tran, D. N.; Battilocchio, C.; Sedelmeier, J.; Sedelmeier, G.; Schenkel, B.; Ley, S. V. Angew. Chem., Int. Ed. 2016, 55, 14085.

[23] Minisci, F.; Bernardi, R.; Bertini, F.; Galli, R.; Perchinummo, M. Tetrahedron 1971, 27, 3575 .

[24] Proctor, R. S. J.; Phipps, R. J. Angew. Chem., Int .Ed. 2019, 58, 13666

[25] Li, G. X.; Morales-Rivera, C. A.; Wang, Y. X.; Gao, F.; He, G.; Liu, P.; Chen, G. Chem. Sci. 2016, 7, 6407.

[26] Zhang, W. M.; Dai, J. J.; Xu, J.; Xu, H. J. J. Org. Chem. 2017, 82, 2059.

[27] Matsui, J. K.; Primer, D. N.; Molander, G. A. Chem. Sci. 2017, 8 , 3512.

[28] Yan, H.; Hou, Z. W.; Xu, H. C. Angew. Chem., Int. Ed. 2019, 58, 4592.

[29] Amani, J.; Sodagar, E.; Molander, G. A. Org. Lett. 2016, 18, 732.

[30] Amani, J.; Molander, G. A. J. Org. Chem. 2017, 82, 1856.

[31] Amani, J.; Alam, R.; Badir, S.; Molander, G. A. Org. Lett. 2017, 19, 2426.

[32] Amani, J.; Molander, G. A. Org. Lett. 2017, 19, 3612.

[33] Stache, E. E.; Rovis, T.; Doyle, A. G. Angew. Chem., Int. Ed. 2017, $56,3679$.

[34] Liu, W.; Liu, P.; Lv, L.; Li, C. J. Angew. Chem., Int. Ed. 2018, 57, 13499.

[35] Huang, H.; Jia, K.; Chen, Y. Angew. Chem., Int. Ed. 2015, 54, 1881.

[36] Miyazawa, K.; Yasu, Y.; Koike, T.; Akita, M. Chem. Commun. 2013, 49, 7249.

[37] Miyazawa, K.; Koike, T.; Akita, M. Adv. Synth. Catal. 2014, 356 2749.

[38] Li, Y.; Miyazawa, K.; Koike, T.; Akita, M. Org. Chem. Front. 2015 2,319 .

[39] Chinzei, T.; Miyazawa, K.; Yasu, Y.; Koike, T.; Akita, M. RSC Adv. 2015, 5, 21297.

[40] Iwata, Y.; Tanaka, Y.; Kubosaki, S.; Morita, T.; Yoshimi, Y. Chem Commun. 2018, 54, 1257.

[41] Lang, S. B.; Wiles, R. J.; Kelly, C. B.; Molander, G. A. Angew. Chem., Int. Ed. 2017, 56, 15073.

[42] Huo, H.; Harms, K.; Meggers, E. J. Am. Chem. Soc. 2016, 138 , 6936.

[43] Tutkowski, B.; Meggers, E.; Wiest, O. J. Am. Chem. Soc. 2017, $139,8062$.

[44] Plasko, D. P.; Jordan, C. J.; Ciesa, B. E.; Merrill, M. A.; Hanna, J. M. Photochem. Photobiol. Sci. 2018, 17, 534.

[45] Yi, J.; Badir, S. O.; Alam, R.; Molander, G. A. Org. Lett. 2019, 21, 4853.

[46] Yu, X. Y.; Zhou, Q. Q.; Wang, P. Z.; Liao, C. M.; Chen, J. R.; Xiao, W. J. Org. Lett. 2018, 20, 421.

[47] Liu, M.; Huang, H.; Chen, Y. Chin. J. Chem. 2018, 36, 1209.

[48] Xie, S.; Li, D.; Huang, H.; Zhang, F.; Chen, Y. J. Am. Chem. Soc. 2019, 141, 16237.

[49] Li, X.; Han, M.-Y.; Wang, B.; Wang, L.; Wang, M. Org. Biomol. Chem. 2019, 17, 6612.

[50] Sim, J.; Campbell, M. W.; Molander, G. A. ACS Catal. 2019, 9, 1558.

[51] Campbell, M. W.; Compton, J. S.; Kelly, C. B.; Molander, G. A. J. Am. Chem. Soc. 2019, 141, 20069.

[52] Lima, F.; Sharma, U. K.; Grunenberg, L.; Saha, D.; Johannsen, S.; Sedelmeier, J.; Van der Eycken, E. V.; Ley, S. V. Angew. Chem., Int. Ed. 2017, 56, 15136.

[53] Lima, F.; Grunenberg, L.; Rahman, H. B. A.; Labes, R.; Sedelmeier, J.; Ley, S. V. Chem. Commun. 2018, 54, 5606.

[54] Chen, Y.; May, O.; Blakemore, D. C.; Ley, S. V. Org. Lett. 2019, 21 , 6140 .

[55] Ye, H.; Ye, Q.; Cheng, D.; Li, X.; Xu, X. Tetrahedron Lett. 2018, 59, 2046.

[56] Ye, H.; Zhao, H.; Ren, S.; Ye, H.; Cheng, D.; Li, X.; Xu, X. Tetra- 
hedron Lett. 2019, 60, 1302.

[57] Shu, C.; Noble, A.; Aggarwal, V. K. Angew. Chem., Int. Ed. 2019, $58,3870$.

[58] Kaiser, D.; Noble, A.; Fasano, V.; Aggarwal, V. K. J. Am. Chem. Soc. 2019, 141, 14104.

[59] Yasu, Y.; Koike, T.; Akita, M. Adv. Synth. Catal. 2012, 354, 3414.

[60] Zou, Y. Q.; Chen, J. R.; Liu, X. P.; Lu, L. Q.; Davis, R. L.; Jorgensen, K. A.; Xiao, W. J. Angew. Chem., Int. Ed. 2012, 51, 784.

[61] Weng, W. Z.; Liang, H.; Zhang, B. Org. Lett. 2018, 20, 4979.
[62] Liu, T.; Li, Y.; Lai, L.; Cheng, J.; Sun, J.; Wu, J. Org. Lett. 2018 20, 3605.

[63] Ye, S.; Li, X.; Xie, W.; Wu, J. Asian J. Org. Chem. 2019, 8, 893

[64] Liu, T.; Ding, Y.; Fan, X.; Wu, J. Org. Chem. Front. 2018, 5, 3153.

[65] Gong, X.; Yang, M.; Liu, J.-B.; He, F.-S.; Wu, J. Org. Chem. Front. 2020, 7, 938.

[66] Clausen, F.; Kischkewitz, M.; Bergander, K.; Studer, A. Chem. Sci. 2019, 10, 6210 .

(Cheng, F.) 